UCRL-TR-203892

\title{
How Accurately can we Calculate Thermal Systems?
}

\author{
by \\ Dermott E. Cullen, LLNL \\ Roger N. Blomquist, ANL \\ Chris Dean, Serco Assurance \\ Dave Heinrichs, LLNL \\ Mikhail A. Kalugin, Kurchatov Institute \\ Mark Lee, DOE/LSO \\ Yi-Kang Lee, CEA/Saclay \\ Robert MacFarlane, LANL \\ Yasunobu Nagaya, JAERI \\ Andrej Trkov, IAEA \\ Contact \\ Dermott E. Cullen \\ University of California \\ Lawrence Livermore National Laboratory \\ P.O.Box 808/L-159 \\ Livermore, CA 94550
}

Tele: 925-423-7359

E.Mail: cullen1@llnl.gov

U.S. Department of Energy

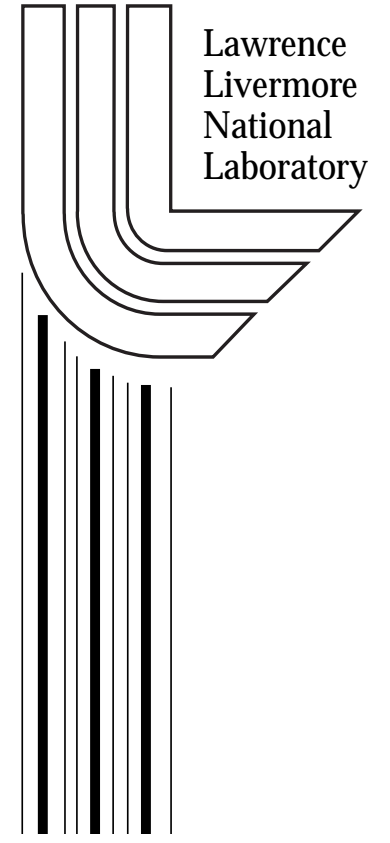

April 20, 2004

Approved for public release; further dissemination unlimited. 


\section{DISCLAIMER}

This document was prepared as an account of work sponsored by an agency of the United States Government. Neither the United States Government nor the University of California nor any of their employees, makes any warranty, express or implied, or assumes any legal liability or responsibility for the accuracy, completeness, or usefulness of any information, apparatus, product, or process disclosed, or represents that its use would not infringe privately owned rights. Reference herein to any specific commercial product, process, or service by trade name, trademark, manufacturer, or otherwise, does not necessarily constitute or imply its endorsement, recommendation, or favoring by the United States Government or the University of California. The views and opinions of authors expressed herein do not necessarily state or reflect those of the United States Government or the University of California, and shall not be used for advertising or product endorsement purposes.

Work performed under the auspices of the U. S. Department of Energy by the University of California Lawrence Livermore National Laboratory under Contract W-7405-Eng-48.

This report has been reproduced directly from the best available copy.

Available to DOE and DOE contractors from the

Office of Scientific and Technical Information

P.O. Box 62, Oak Ridge, TN 37831

Prices available from (423) 576-8401

http://apollo.osti.gov/bridge/

Available to the public from the National Technical Information Service

U.S. Department of Commerce 5285 Port Royal Rd., Springfield, VA 22161 http://www.ntis.gov/

OR

Lawrence Livermore National Laboratory Technical Information Department's Digital Library http://www.llnl.gov/tid/Library.html 
UCRL-TR-203892

\author{
How Accurately can we \\ Calculate Thermal Systems? \\ by \\ Dermott E. Cullen, LLNL \\ Roger N. Blomquist, ANL \\ Chris Dean, Serco Assurance \\ Dave Heinrichs, LLNL \\ Mikhail A. Kalugin, Kurchatov Institute \\ Mark Lee, DOE/LSO \\ Yi-Kang Lee, CEA/Saclay \\ Robert MacFarlane, LANL \\ Yasunobu Nagaya, JAERI \\ Andrej Trkov, IAEA \\ Contact \\ Dermott E. Cullen \\ University of California \\ Lawrence Livermore National Laboratory \\ P.O.Box 808/L-159 \\ Livermore, CA 94550
}

Tele: 925-423-7359

E.Mail: cullen1@llnl.gov

Website: http://www.llnl.gov.cullen1

\title{
Overview
}

April 20, 2004

I would like to determine how accurately a variety of neutron transport code packages (code and cross section libraries) can calculate simple integral parameters, such as Keff, for systems that are sensitive to thermal neutron scattering. Since we will only consider theoretical systems, we cannot really determine absolute accuracy compared to any real system. Therefore rather than accuracy, it would be more precise to say that I would like to determine the spread in answers that we obtain from a variety of code packages. This spread should serve as an excellent indicator of how accurately we can really model and calculate such systems today. Hopefully, eventually this will lead to improvements in both our codes and the thermal scattering models that they use in the future.

In order to accomplish this I propose a number of extremely simple systems that involve thermal neutron scattering that can be easily modeled and calculated by a variety of neutron transport codes. These are theoretical systems designed to emphasize the effects of thermal scattering, since that is what we are interested in studying. I have attempted to 
keep these systems very simple, and yet at the same time they include most, if not all, of the important thermal scattering effects encountered in a large, water-moderated, uranium fueled thermal system, i.e., our typical thermal reactors.

\section{Acknowledgements}

I thank Ernest Plechaty for contributing interesting and informative discussions of thermal scattering. I thank Enrico Sartori for spreading the word about this comparison, and contacting a number of people who eventually contributed to this comparison.

\section{Ground rules}

I want to test each code package completely, including the important, and yet often overlooked influence of code users on code results. In order to do this I ask each participant to assume they are the local expert on a code. Someone comes to your office and asks you what your best estimate is of K-eff for a system, using thermal scattering law data or free atom scattering. They are not experts on neutron transport or your code, so they only define the geometry and materials. You, as the local code expert, must then make all decisions as far as what nuclear data to use and what input parameters to define for your code, and supply the requested K-eff. If you routinely use more than one nuclear data library, or would like to show results using a variety of input options, feel free to send more than one set of results using each of your data libraries or input options; in this case please clearly state what data and input options were used for each set of results, so that we can distinguish between your sets of results.

\section{My Simplest Possible Infinite Repeating Lattice of Uranium/Water Cells}

To simulate a water-moderated, uranium fueled, thermal reactor, we can use a simple cylindrical uranium pin, centered in and surrounded by a square cell filled with water. To simulate an infinite array of cells we make the four sides of the square totally reflecting, i.e., no leakage. The third dimension of the cell, along the axis of the cylinder, can be either infinite in extent, or finite with reflecting surfaces, whatever is easiest for your code to handle. The net effect is a system that is infinite in all directions, so there is no leakage, and infinitely repeats the cylindrical pin surrounded by water. As far as K-eff is concerned, in this system we need only be concerned with neutron production and absorption; again, there is no leakage.

Below I illustrate a $1 / 4 "(0.635 \mathrm{~cm})$ radius uranium cylinder (the red zone), surrounded by a $2 "(5.08 \mathrm{~cm})$ square filled with water (the green zone). For our use here it is sufficient to maintain the 2" pitch, as well as the density of fuel and water, and ONLY vary the radius of the pin, and $U^{235}$ to $U^{238}$ ratio to make the system near critical, i.e., $\mathrm{K}$ eff $\sim 1.0$, using Free Atom thermal scattering. In each case we will then change only one input parameter to use either Free Atom or $\mathrm{S}(\alpha, \beta)$ thermal scattering law data. In this manner we can be sure that any differences in K-eff and neutron spectrum are due ONLY to the difference in the thermal scattering model used. 


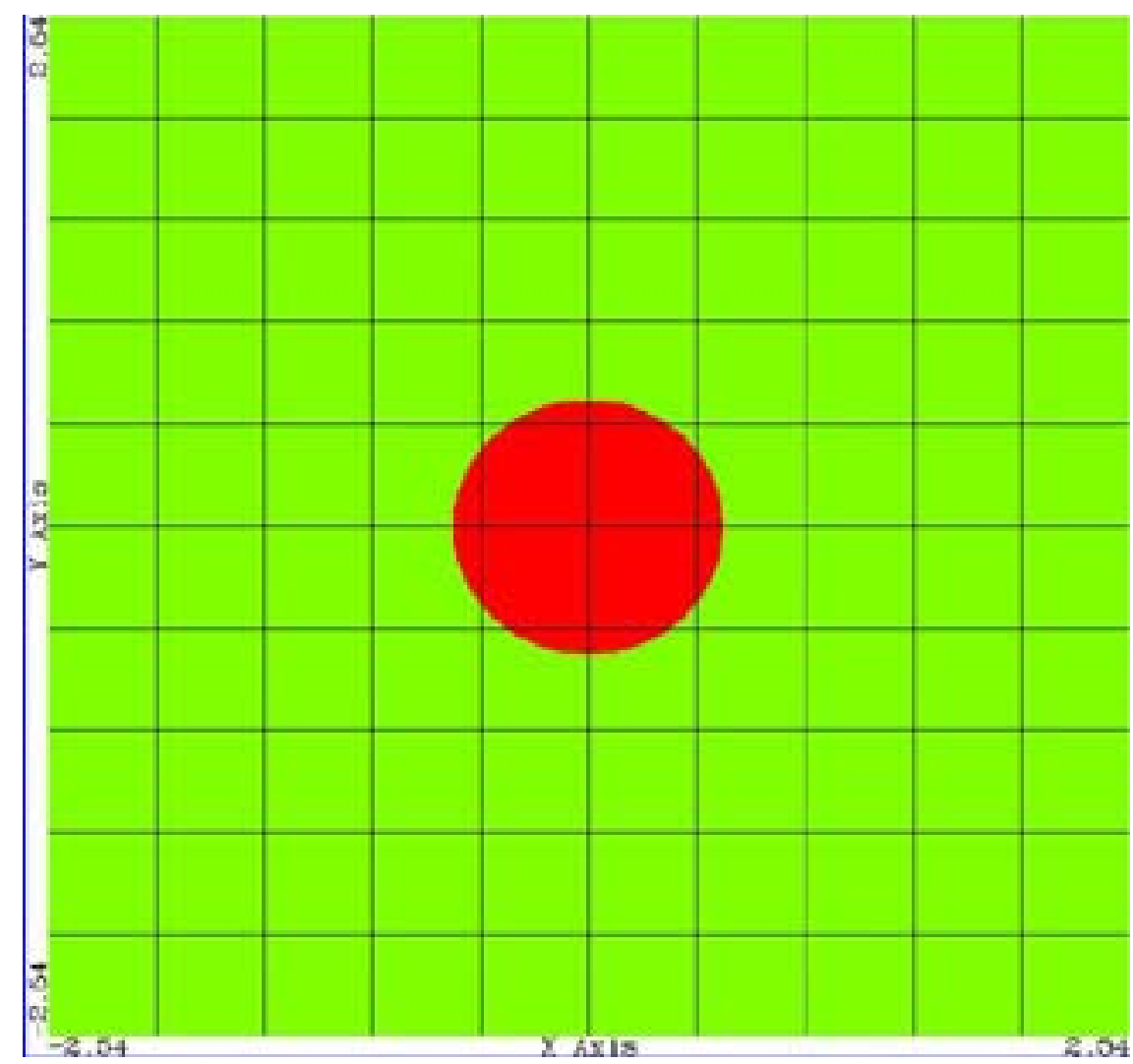

Fig. 1: 1/4" radius fuel pin, in 2" square water cell

I propose three different pin cell problems, and for each you are asked to estimate K-eff using thermal scattering law data or free atom data; therefore there are a total of six problems. In all cases the problem is a square 2" $(5.08 \mathrm{~cm})$ cell filled with water at 1.0 grams/cc density, containing a cylindrical fuel pin at 18.8 grams/cc density, and a radius of $1 / 2 ", 1 / 4$ " or $1 / 8$ ", where the $\mathrm{U}^{235}$ and $\mathrm{U}^{238}$ ratio has been varied to make the system near critical using free atom data. Results should be calculated using both free atom and $\mathrm{S}(\alpha, \beta)$ thermal scattering law models.

Please remember that these are only theoretical problems that simulate the effects we are interested in. For simplicity in analyzing results the fuel is composed only of $\mathrm{U}^{235}$ and $\mathrm{U}^{238}$, and there is no cladding or air gap. The ratio of water to fuel has been defined to maximize the effect of thermal scattering, since this is the effect we are interested in.

I ask that contributors submit answers for these problem, and only these problems, so that they can be meaningfully compared to answers from other codes. Please, do not try to be creative and make changes to these specifications; unless your answers correspond to exactly these problems we cannot use your answers.

Contributors can send any number of sets of results; we are particularly interested in results using different nuclear data libraries or input options with the same transport code. 
It is worth noting that the test cases used here are not exactly representative of commercial power reactors where the pitch is typically about $1.25 \mathrm{~cm}=\sim 0.5$ " and pin radius less than $.5 \mathrm{~cm}=\sim 0.2$ ". Such systems are somewhat less sensitive to the thermal scattering law data. Since the primary topic of this paper is the effect of thermal scattering, we have defined theoretical systems in which the effects of thermal scattering are maximized; the test cases here are similar, but not identical to actual commercial power reactors.

\section{All three problem include water surrounding the central fuel pin}

2 " square water

1.0 grams/cc density

2.0 atoms of hydrogen to 1.0 atoms of oxygen

Below I describe each of the three problems, highlighting how they differ from one another.

\section{Problem \#1}

$\mathbf{1} / 2 ”(1.27 \mathbf{~ c m})$ radius fuel pin - 18.8 grams/cc density - total $<v>$ - static criticality

\section{Problem \#2}

$\mathbf{1 / 4}$ ” $(0.635 \mathrm{~cm})$ radius fuel pin -

99.02 atoms of $U^{238}$ to $\mathbf{0 . 9 8}$ atoms of $\mathrm{U}^{235}$

Problem \#3

1/8” $(0.3175 \mathrm{~cm})$ radius fuel pin -

18.8 grams/cc density - total $<v>-$ static criticality
96.5 atoms of $\mathrm{U}^{238}$ to 3.5 atoms of $\mathrm{U}^{235}$

18.8 grams/cc density - total $\langle v\rangle$ - static criticality

$\mathbf{3 0 . 0}$ atoms of $\mathrm{U}^{238}$ to $\mathbf{7 0 . 0}$ atoms of $\mathrm{U}^{235}$

\section{Basic Definitions}

When I was in graduate school one of my professors said that there are more definitions of reactor parameters than fleas on a dog. I had completely forgotten about this statement until I started this study. Even for something as seemingly simple as K-eff the various codes uses different definitions, differing mostly in how they handle non-fission, multiple neutron emission, such as $(n, 2 n),(n, 3 n)$, etc. These different definitions of K-eff lead quite naturally to different definitions of quantities such as the median fission energy, neutron lifetime, or removal time, as well as different production, absorption and leakage energy dependent spectra. I'll try to explain the differences in the appendix. For now it is sufficient for the reader to know that differences in the basic definitions of K-eff will lead to slightly different values of K-eff even if we are using deterministic codes where there are no statistical uncertainties. At least for this study these differences are small compared to the differences that we see in values of K-eff calculated by each code, so that for our comparison of integral parameters we need not be concerned in this paper.

\section{Using Thermal Scattering Law Data}

You might think that it would be sufficient if a code designer uniquely defines the basic data they used, as for example by saying: I used the ENDF/B-VI thermal scattering law 
data, but it isn't. One interesting result of this comparison was to discover how many different interpretations there are of the same basic data in each code. Some codes sample $\mathrm{S}(\alpha, \beta)$ data directly, some convert it to continuous double differential data in secondary energy and scattering cosine, some convert it to discrete data in secondary energy and direction, and some use a multi-group representation. Our results indicate that all of these representations can accurately reproduce a simple integral parameter, such as K-eff. It remain to be seen whether or not the detailed differences in the energy dependent neutron flux effect other parameters of interest; this is outside the scope of the current study, but suggests possible follow-on studies; see the appendix.

\section{Bound versus Free Atom}

In this report we present results using two different models of thermal scattering. The first model uses thermal scattering law data, $\mathrm{S}(\alpha, \beta)$. With this model thermal scattering can include atomic translational motion as well as vibration and rotation. The second model uses free atoms, in which we assume the thermal atomic motion is Maxwellian. Both bound and free models include a priori and a posteriori affects. The a priori affect is that the thermal motion changes the relative speed between target atoms and incident neutrons, which means that the reaction rate is changed; this effect is accounted for by Doppler broadening the cross sections, e.g., see the below plot of the hydrogen cross section that show the affect of Doppler broadening. The a posteriori affect is that the thermal motion changes the secondary direction and speed of scattered neutrons; this is where the bound and free models really differ. In order to correctly calculate our example problems a priori and a posteriori affects must both be included in the neutron transport calculation. For purposes of this report we define the terms free atom and free gas to be synonymous; we assume that both refer to a model in which atoms have a thermal motion that can be described by a Maxwellian; this motion is used both a priori to Doppler broaden cross sections and a posteriori to describe the distribution of secondary neutrons in direction and energy.

\section{Round Zero Results}

Of the many initial results received for this study over $30 \%$ were in error. Almost all of these errors could be tracked to incorrect input parameters or data used with the codes. In some cases even code authors, who should be most familiar with their own codes, used incorrect input parameters or data. Based on these Round Zero results the first point that we can learn from this study is that it is not at all trivial to correctly use these codes and obtain reliable answers. Users should use care and if at all possible compare the results from several codes, as we have done in this study. In addition, code designers should try to make their codes as user friendly as possible, and provide adequate documentation to assist users in preparing input for their codes, and to assist them in interpreting output results.

This study is not intended to needlessly embarrass anyone, so that all contributors were given the opportunity to correct their round zero results, before publication of this report. 
Results from two codes were withdrawn. All of the other contributors corrected their results making them suitable for publication, as the Round One Results presented below.

Later in this report contributors are also given the opportunity to help their code users avoid difficulties in using their code by explaining errors they made in using their codes; this is strictly voluntary.

\section{Round One Results}

Round One Results suitable for publication are included here for the following codes and participants,

$\begin{array}{ll}\text { COG } & \text { Dave Heinrichs and Mark Lee } \\ \text { KENO } & \text { Dave Heinrichs } \\ \text { MCNP } & \text { Bob MacFarlane, Mark Lee, Andrej Trkov, Dave Heinrichs, Red Cullen } \\ \text { MCU } & \text { Mikhail Kalugin } \\ \text { MONK } & \text { Chris Dean } \\ \text { MVP } & \text { Yasunobu Nagaya } \\ \text { TART } & \text { Red Cullen and Mark Lee } \\ \text { TRIPOLI-4.3 Yi-Kang Lee } \\ \text { VIM } & \text { Roger Blomquist and Dave Heinrichs } \\ \text { WIMS } & \text { Dave Heinrichs and Andrej Trkov }\end{array}$

It is important for the reader to understand that we make no claim that the results from any one code presented here are any better, or worse, than the results from any other code. The results presented here are simply what the codes produce today; please do not read more into these results. Please remember that the intent of this study is to determine the spread in results produced by a variety of codes, from which we can get a good idea of how reliable the results are from any one given code. It is not the intent of this study to crown any code to be the winner of some contest. Hopefully based on the results presented here all of the codes will be winners, in the sense that these results can be used by all of the codes to even further improve their results.

In the below table "free" results correspond to using free atom data, and "bound" results correspond to using thermal scattering law data, e.g., $\mathrm{S}(\alpha, \beta)$. All of the participating codes contributed results using thermal scattering law data, e.g., $\mathrm{S}(\alpha, \beta)$. Some codes either cannot perform calculations using free atom hydrogen or chose not to submit free atom results, as indicated by no "free" results for these codes. 
K-eff for Monte Carlo Codes

\begin{tabular}{|c|c|c|c|c|c|c|c|}
\hline $\begin{array}{c}\text { Case } \\
\#\end{array}$ & Code & $\begin{array}{l}1 / 2 " \\
\text { Free }\end{array}$ & $\begin{array}{c}1 / 2 " \\
\text { Bound }\end{array}$ & $\begin{array}{l}1 / 4 " \\
\text { Free }\end{array}$ & $\begin{array}{c}1 / 4 " \\
\text { Bound }\end{array}$ & $\begin{array}{l}1 / 8^{\prime \prime} \\
\text { Free }\end{array}$ & $\begin{array}{c}1 / 8^{\prime \prime} \\
\text { Bound }\end{array}$ \\
\hline 1 & COG (Mark) & $1.0112(12)$ & $0.9636(12)$ & $1.0111(12)$ & $0.9159(12)$ & $1.0109(12)$ & $0.9019(12)$ \\
\hline 2 & COG (Dave) & 1. $0115(12)$ & $0.9608(12)$ & $1.0121(12)$ & $0.9148(12)$ & 1. $0133(12)$ & $0.9065(12)$ \\
\hline 3 & KENO (Dave) & $1.0092(38)$ & $0.9628(5)$ & $1.0133(7)$ & $0.9163(7)$ & $1.0133(8)$ & $\odot .9050(7)$ \\
\hline 4 & MCNP5 (Bob) & $1.01283(12)$ & $0.96062(13)$ & $1.01078(16)$ & $\odot .91221(18)$ & $1.01206(22)$ & $0.89867(23)$ \\
\hline 5 & MCNP5 (Mark) & $1.01236(38)$ & $0.96073(42)$ & $1.01015(52)$ & ๑.91115(55) & $1.01200(68)$ & $0.89911(70)$ \\
\hline 6 & MCNP5 (Red) & $1.01294(40)$ & $0.96059(42)$ & $1.01133(56)$ & $0.91204(58)$ & $1.01299(74)$ & $0.89932(73)$ \\
\hline 7 & MCNP5 (Red) & $1.01298(13)$ & $0.96046(14)$ & $1.01089(17)$ & $0.91208(18)$ & $1.01187(23)$ & $0.89854(23)$ \\
\hline 8 & MCNP5 (Red) & $1.01279(4)$ & $\odot .96067(4)$ & $1.01094(6)$ & $\odot .91212(6)$ & 1. $01187(7)$ & $\odot .89882(8)$ \\
\hline 9 & MCNP4C (Dave) & $1.0101(05)$ & $0.9597(06)$ & $1.0105(08)$ & $0.9133(08)$ & 1.0103(09) & $0.9027(11)$ \\
\hline 10 & MCNP4B (Andrej) & $1.01071(6)$ & $0.96061(6)$ & $1.00932(9)$ & ๑.91380(9) & $1.01038(11)$ & $0.90201(11)$ \\
\hline 11 & $\operatorname{MCNPX} 21$ (Andrej) & 1. $01075(6)$ & $0.96071(7)$ & $1.00927(8)$ & $0.91402(9)$ & $1.01023(11)$ & $0.90199(11)$ \\
\hline 12 & $\operatorname{MCNPX} 24($ Andrej $)$ & - - & $0.96071(6)$ & - - & - - & - - & - \\
\hline 13 & MCNPX24(Andrej) & $1.01292(6)$ & ๑.96211(7) & $1.01100(9)$ & $0.91496(9)$ & $1.01178(11)$ & $\odot .90264(11)$ \\
\hline 14 & $\operatorname{MCNPX} 24($ Andre $j)$ & $1.01292(6)$ & $\odot .96055(6)$ & $1.01100(9)$ & $0.91207(9)$ & $1.01178(11)$ & $0.89874(12)$ \\
\hline 15 & MCNPX24(Andrej) & $1.01292(6)$ & $0.96044(6)$ & $1.01100(9)$ & $\odot .91167(9)$ & $1.01178(11)$ & $0.89850(11)$ \\
\hline 16 & MCU(Mikhail) & - - & $0.96284(35)$ & - - & $0.91454(50)$ & -1 & $\odot .89653(56)$ \\
\hline 17 & MCU(Mikhail) & $1.01680(30)$ & ๑.96378(30) & $1.01632(40)$ & ๑.91404(40) & $1.01556(40)$ & $0.89842(30)$ \\
\hline 18 & MONK8B(Chris) & 1. $01300(5)$ & $0.95990(5)$ & $1.01130(5)$ & $0.91140(5)$ & $1.0132(2)$ & $0.8991(1)$ \\
\hline 19 & MONK9 (Chris) & $1.0136(3)$ & $0.9593(3)$ & $1.0117(2)$ & $0.9101(2)$ & $1.0121(2)$ & $0.8969(1)$ \\
\hline 20 & MVP(Yasunobu) & $1.01299(8)$ & $0.96065(9)$ & $1.01169(12)$ & $0.91309(15)$ & $1.01282(17)$ & $0.90016(19)$ \\
\hline 23 & TARTO4(Red) & $1.00916(50)$ & $0.96114(50)$ & $1.00983(50)$ & ๑.91548(50) & $1.01072(50)$ & $0.90333(50)$ \\
\hline 24 & TARTO4 (Red) & $1.00952(5)$ & $0.96071(5)$ & $1.00933(5)$ & $0.91544(5)$ & $1.01091(5)$ & $0.90293(5)$ \\
\hline 25 & TARTO4(Mark) & $1.0101(4)$ & $0.9598(5)$ & $1.0098(7)$ & $0.9138(8)$ & $1.0126(10)$ & $0.9007(11)$ \\
\hline 26 & TARTO4 (Mark) & $1.00960(4)$ & $0.96073(4)$ & $1.00926(7)$ & $0.91537(8)$ & $1.01097(10)$ & $0.90312(11)$ \\
\hline 27 & TRIPOLI4(Yi) & $1.01295(11)$ & $0.96046(10)$ & $1.01243(10)$ & $0.91197(10)$ & $1.01448(11)$ & $0.89766(10)$ \\
\hline $\begin{array}{l}21 \\
28\end{array}$ & VIM(Dave) & $1.0157(8)$ & $0.9609(8)$ & $1.0150(09)$ & $0.9131(09)$ & $1.0168(11)$ & $0.9020(11)$ \\
\hline \multirow[t]{5}{*}{$\begin{array}{l}20 \\
29\end{array}$} & VIM(Roger) & $1.00525(7)$ & $0.95939(7)$ & $1.00680(9)$ & $0.90877(9)$ & $1.03463(10)$ & $0.89629(10)$ \\
\hline & Average & 1.011792 & 0.960915 & 1.011046 & 0.913212 & 1.013173 & 0.900503 \\
\hline & Minimum & 1.005250 & 0.959300 & 1.006800 & 0.908770 & 1.010230 & 0.896290 \\
\hline & Maximum & 1.016800 & 0.963780 & 1.016320 & 0.916300 & 1.034630 & 0.906500 \\
\hline & Spread & 0.011550 & 0.004480 & 0.009520 & 0.007530 & 0.024400 & 0.010210 \\
\hline
\end{tabular}

All of these codes performed very well, based on the excellent agreement that we see. If I assume all of the above Monte Carlo codes are equally good, these results say that if I randomly select one of them to use for these calculations, the spread in the answer I get for K-eff would amount to over $2 \%$ for free and $1 \%$ for bound.

Multiple results for the same code are quite interesting, because they clearly show that the code user can influence results by selecting code options and nuclear data that can have a large impact on results, e.g., we see differences for the same code at the $1 \%$ level.

It is also interesting to have multiple results from the same code using different convergence criteria or number of sample neutrons; these results allow us to test the validity of the uncertainty output by the codes, e.g., are multiple results all consistent as far as value and uncertainty? Results seem to indicate that it is more complicated than this, again, where user selected options can influence results, such as deciding how many settle cycles to use before starting to actually accumulate results to define K-eff. 
K-eff for deterministic Codes

\begin{tabular}{|c|c|c|c|c|c|c|c|}
\hline $\begin{array}{c}\text { Case } \\
\#\end{array}$ & Code & $\begin{array}{c}1 / 2 " \\
\text { Free }\end{array}$ & $\begin{array}{c}1 / 2 " \\
\text { Bound }\end{array}$ & $\begin{array}{c}1 / 4 " \\
\text { Free }\end{array}$ & $\begin{array}{c}1 / 4 " \\
\text { Bound }\end{array}$ & $\begin{array}{c}1 / 8^{\prime \prime} \\
\text { Free }\end{array}$ & $\begin{array}{c}1 / 8^{\prime \prime} \\
\text { Bound }\end{array}$ \\
\hline 30 & WIMSD5B(Andrej) & 1.01042 & 0.96226 & 1.00793 & 0.91465 & 1.01194 & 0.90620 \\
31 & WIMSD5B(Andrej) & 1.01037 & 0.96222 & 1.00792 & 0.91464 & 1.00680 & 0.90025 \\
32 & WIMSD5B(Andrej) & 1.01037 & 0.96239 & 1.00792 & 0.91551 & 1.00680 & 0.90242 \\
33 & WIMSD5B(Andrej) & -- & 0.96196 & -- & 0.91560 & -- & 0.90791 \\
34 & WIMSANL(Dave) & -- & 0.95948 & -- & 0.90877 & -- & 0.88161 \\
& & & & & & \\
& Average & 1.010387 & 0.961662 & $\mathbf{1 . 0 0 7 9 2 3}$ & $\mathbf{0 . 9 1 3 8 3 4}$ & $\mathbf{1 . 0 0 8 5 1 3}$ & $\mathbf{0 . 8 9 9 6 7 8}$ \\
& Minimum & $\mathbf{1 . 0 1 0 3 7 0}$ & $\mathbf{0 . 9 5 9 4 8 0}$ & $\mathbf{1 . 0 0 7 9 2 0}$ & $\mathbf{0 . 9 0 8 7 7 0}$ & $\mathbf{1 . 0 0 6 8 0 0}$ & $\mathbf{0 . 8 8 1 6 1 0}$ \\
& Maximum & $\mathbf{1 . 0 1 0 4 2 0}$ & $\mathbf{0 . 9 6 2 3 9 0}$ & $\mathbf{1 . 0 0 7 9 3 0}$ & $\mathbf{0 . 9 1 5 6 0 0}$ & $\mathbf{1 . 0 1 1 9 4 0}$ & $\mathbf{0 . 9 0 7 9 1 0}$ \\
& Spread & $\mathbf{0 . 0 0 0 0 5 0}$ & $\mathbf{0 . 0 0 2 9 1 0}$ & $\mathbf{0 . 0 0 0 0 1 0}$ & $\mathbf{0 . 0 0 6 8 3 0}$ & $\mathbf{0 . 0 0 5 1 4 0}$ & $\mathbf{0 . 0 2 6 3 0 0}$ \\
\hline
\end{tabular}

The WIMS results are quite interesting, first because the WIMSD5B results are in very good agreement with the Monte Carlo results, and second because they clearly illustrate that all versions of supposedly the same code do not give the same answers, as in this case for two variants of the WIMS code: WIMSD5B and WIMSANL. For example, from the $1 / 8$ " bound results we see differences in K-eff of $2.6 \%$.

\section{Summary of Running Conditions}

Below is a brief summary of the running conditions used for each result. Unless otherwise noted the codes used the ENDF/B-VI thermal scattering law data, $\mathrm{S}(\alpha, \beta)$, for hydrogen bound in water. Note the variety of nuclear data libraries used, as well as the variety of settle cycles and batches, requested accuracy, can affect the converged results.

1 COG(Mark) 10,000 batch size; 50 settle cycles; 10,000 batches; ENDF/B-VI,R7

$2 \operatorname{COG}($ Dave) 1,000 batch size; 5 settle cycles; 1, 005 batches; ENDF/B-VI, R7

3 KENO(Dave) 1,000 batch size; 5 settle cycles; 1,005 batches; 238GRPNDF5; Module CSAS25 of SCALE 4.4 used to execute BONAMI, NITAWL-II and KENO-Va.

4 MCNP5(Bob) 10,000 batch size, 50 settle cycles, 1000 batches; ENDF/B-VI.R8

5 MCNP5(Mark) 10,000 batch size; 100 settle cycles; 10,000 batches; ENDF/B-VI, R6

6 MCNP5(Red) 1,000 batch size;100 settle cycles;1000 batches;ENDF/B-VI, R8

7 MCNP5(Red) 10,000 batch size; the rest is the same as above results

8 MCNP5(Red) 100, 000 batch size; the rest is the same as above results

9 MCNP4C(Dave) 1000 batch size; 10 settle cycles; 500 batches; ENDF/B-VI,R2 (.60c)

10 MCNP4B(Andrej) 100, 000 batch size; 20 settle cycles; 400 batches; ENDF/B-VI.R2 (.60c)

11 MCNPX21(Andrej) Same input, same data as 10, running MCNPX-2.1.5 on LinuX

12 MCNPX24(Andrej) Same input, same data as 10, running MCNPX-2.4.0 on Windows

13 MCNPX24(Andrej) ENDF/B-VI.R8 library (locally generated); $\mathrm{s}(\alpha, \beta$ ) from lwtr.01t

14 MCNPX24(Andrej) Same as 13 but $\mathrm{s}(\alpha, \beta)$ file generated locally with 16 angles and 64 bins.

15 MCNPX24(Andrej) Same as 14 but using new $\mathrm{s}(\alpha, \beta)$ from IKE by Margarete Mattes.

$16 \mathrm{MCU}($ Mikhail) $4 \odot$ groups transport approximation for the energy region [ $0-1] \mathrm{eV}$

$17 \mathrm{MCU}(\mathrm{Mikhail})$ pointwise cross sections representation for the energy region [0-4.65] eV

18 MONK8B(Chris)Issued code, 13,193 groups, 100,000 batch size JEF2.2 direct $\mathrm{s}(\alpha, \beta)$ sampling

19 MONK9(Chris) Development code, continuous energy otherwise as above.

20 MVP(Yasunobu) 20,000 batch size; 100 settle cycles; 1000 batches; JENDL-3.3 (293.15K)

23 TART 04 (Red) $0.05 \%$ uncertainty in K-eff; 100 settle; 100, 000 batch size; ENDF/B-VI, R8

24 TARTO4(Red) $0.005 \%$ uncertainty in $\mathrm{K}$-eff; the rest is same as above results

25 TARTO4(Mark) $0.03 \%$ uncertainty in K-eff; 1 , 000 batch size; 100 settle cycles; ENDF/B-VI, R8

26 TARTO4(Mark) $0.003 \%$ uncertainty in K-eff; 10,000 batch size; 100 settle cycles; ENDF/B-VI, R8

27 TRIPOLI4(Yi) 50, 000 batch size; automatic settle cycles; 2000 batches; ENDF/B-VI.R4

28 VIM(Dave) 1,000 batch size; 5 settle cycles; 1,000 batches; ENDF/B-V

29 VIM(Roger)50, 000 batch size; 50 settle cycles; 2, 000 batches; ENDF/B-VI, R8

30 WIMSD5B(Andrej) 172-group ENDF/B-VI, R8, generic library (RI table extrapolation)

31 WIMSD5B(Andrej) 172-group ENDF/B-VI, R8, extended Sig- $\odot$ grid for U-235

32 WIMSD5B(Andrej) 172-group ENDF/B-VI,R8, extended Sig- $\odot$ grid for U-235, new S( $\alpha, \beta$ ) from IKE 
33 WIMSD5B(Andrej) 69-group ENDF/B-VI,R8, generic library (RI table extrapolation)

34 WIMSANL(Dave) 69-group ENDF/B-VI, R7

Below I have selected one result from each code and plotted them versus an arbitrary xaxis; in this case I choose pin radius. The first figure shows results for the three bound cases and the second figure for the three free cases. In each case I show the ratio of the individual results to the average of all results shown on each figure; note, this average is not the same as the average of all results in the above table. Here the spread in results in much smaller than for the above table of all results. For the bound 1/2" radius pin case all of the results agree within only about $+/-0.2 \%$, but for the other pin radii this spread obviously grows to about $+/-0.5 \%$ for the $1 / 8$ " radius pin. For the free cases the agreement is very good with most results are within $+/-0.2 \%$ for all three cases.

Bound Data Pin Cell Results by Code Compared to Average Value

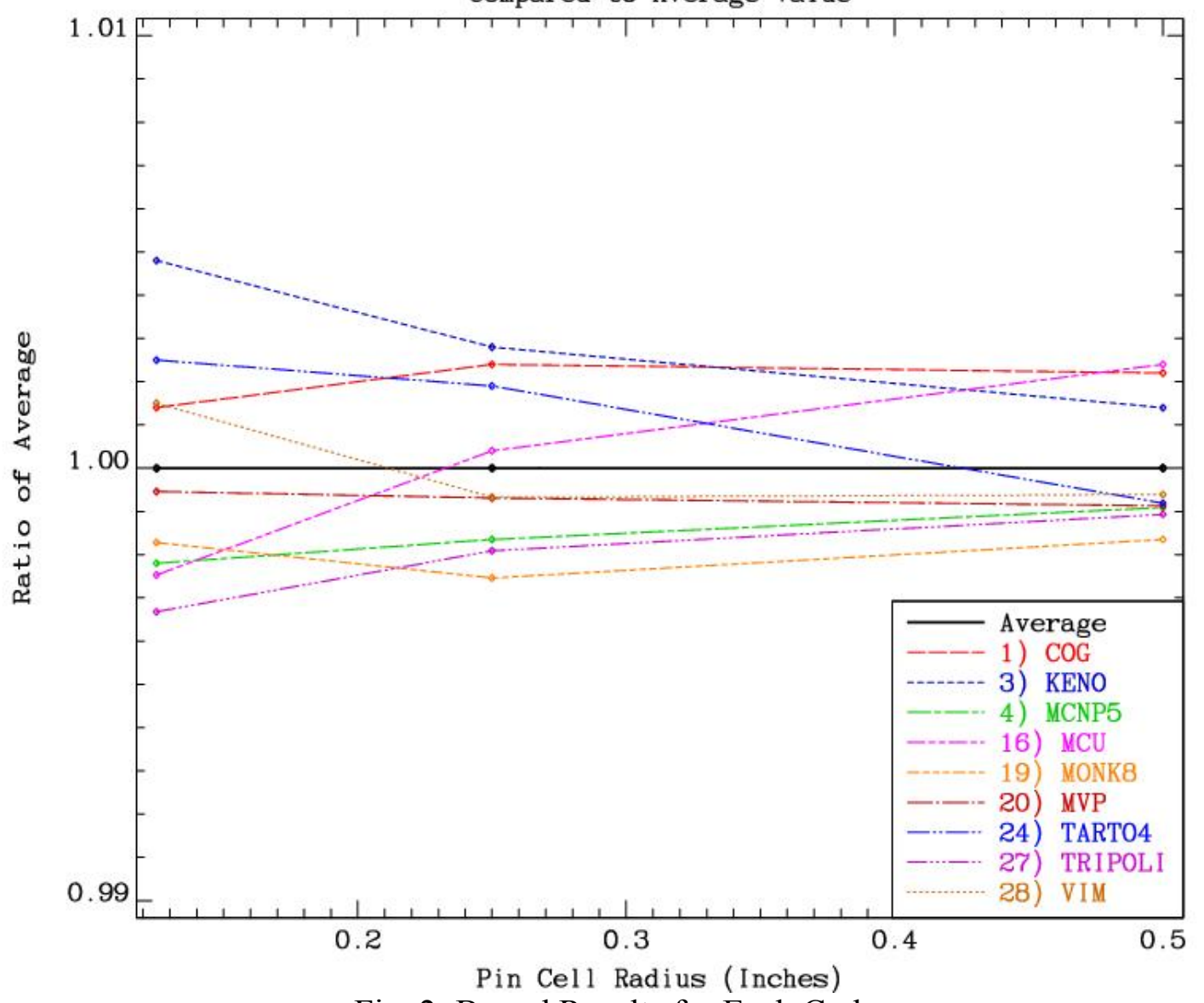

Fig. 2: Bound Results for Each Code 


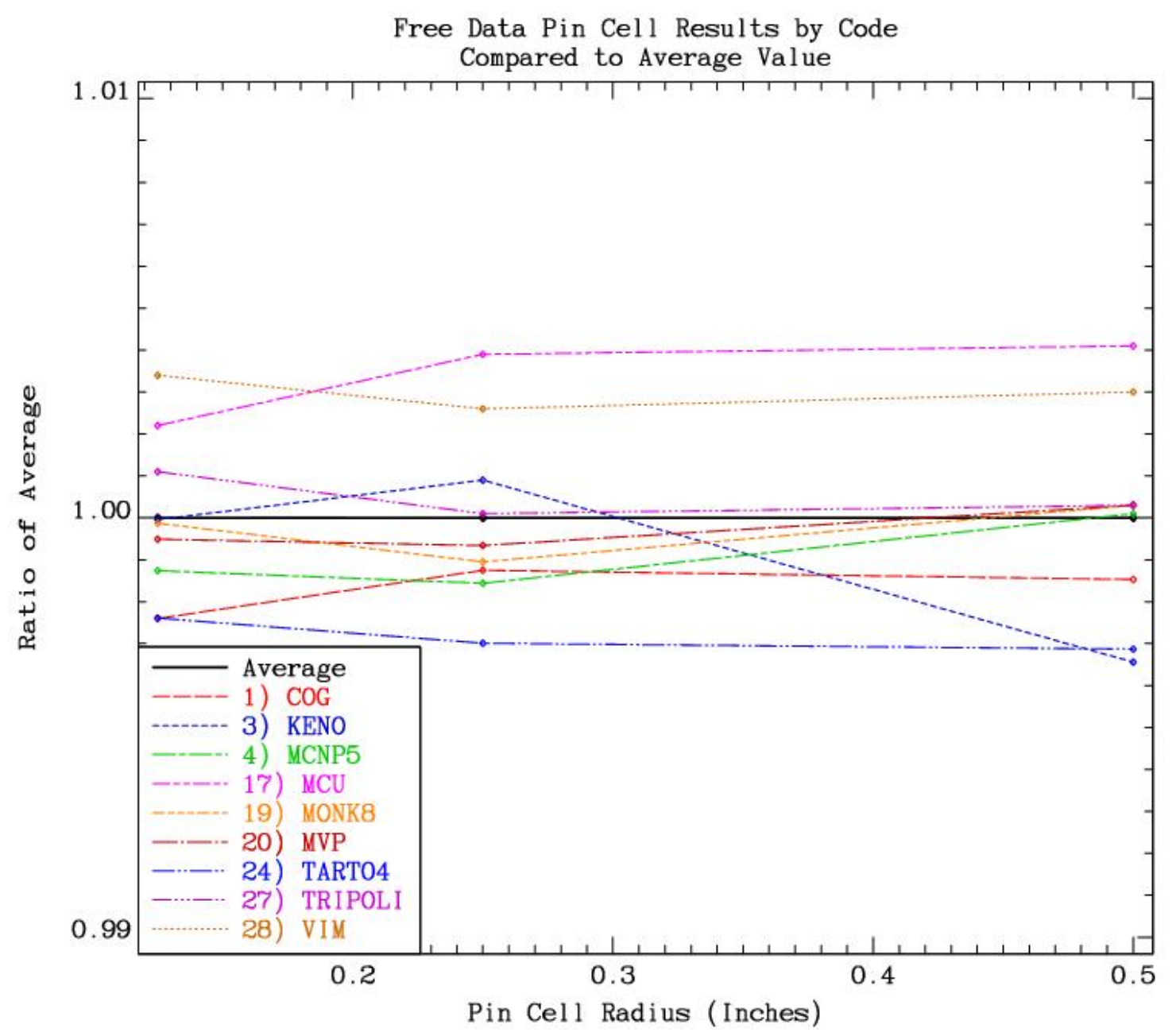

Fig. 3: Free Results for Each Code

\section{The Importance of Free Atom Scattering}

For this study free atom scattering played a vital role in demonstrating how important the effect of bound data is, i.e., if it were not a big effect why we would bother using it? Naturally as long as bound data are available for a given material they should be used, e.g., for these pin cells we would not expect anyone to really model them using free atom hydrogen in water, since bound data are available. But beyond this study free atom scattering plays an important role, in the sense that we now have evaluated data for hundreds of materials, but thermal scattering law data only for a handful of materials. Therefore in real applications we use both thermal scattering law data for materials where they are available, and free atom scattering for all other materials. For example, in these pin cell calculations the results identified as "bound" are really for hydrogen bound in water, plus free atom scattering for oxygen and uranium. Do not make the mistake of assuming the free atom scattering in these other materials does not affect our calculated results. Near the end of this report if you look at fig. 18 you will see the effect of using discrete final energies to represent the bound scattering from hydrogen; that is the narrow spikes in the spectrum. The smooth background between these spikes is due to free atom scattering in the other materials, which makes an important contribution to the overall 
spectrum. To quantitatively illustrate the importance of free atom scattering even in these pin cell problems, I used TART, turning off the free atom scattering for uranium, and oxygen, and hydrogen above $4 \mathrm{eV}$, so that the only thermal scattering was due to the thermal scattering law data below $4 \mathrm{eV}$. This results in a reduction in K-eff of over $0.7 \%$, for a $\mathrm{K}$-eff value which is well outside the range of the results of all codes in table. 1. So make no mistake: accurately modeling free atom scattering is important in real applications.

\section{Difference Between Free and Bound: Median Energy}

Calculation of the median (not average, median) fission energy for these cases helps to explain the difference between the free and bound results. Compared to using free atom scattering, using thermal scattering law data shifts the spectrum to a slightly higher energy, resulting in a less reactive system. We can see this more clearly in the below plots of the spectra.

\section{Detailed Results: Comparison of Spectra}

The below figures show the neutron production spectrum normalized to one neutron removed. The neutron energy is the energy at which the neutron initiated the fission, not the energy of the neutrons emitted by the fission (the later would merely show the induced fission spectrum, from which we would learn very little).

The first figure below shows the production over the entire energy range. Due to the normalization of each spectrum to one neutron removed the production spectra agree at high energy, and only differ at low energies in the $\mathrm{eV}$ range and below due to the difference in the thermal scattering model used.

With the normalization used here, all of the spectra will agree at high energy, so that all of following figures only show the thermal range, so that we can see the differences in greater detail. 


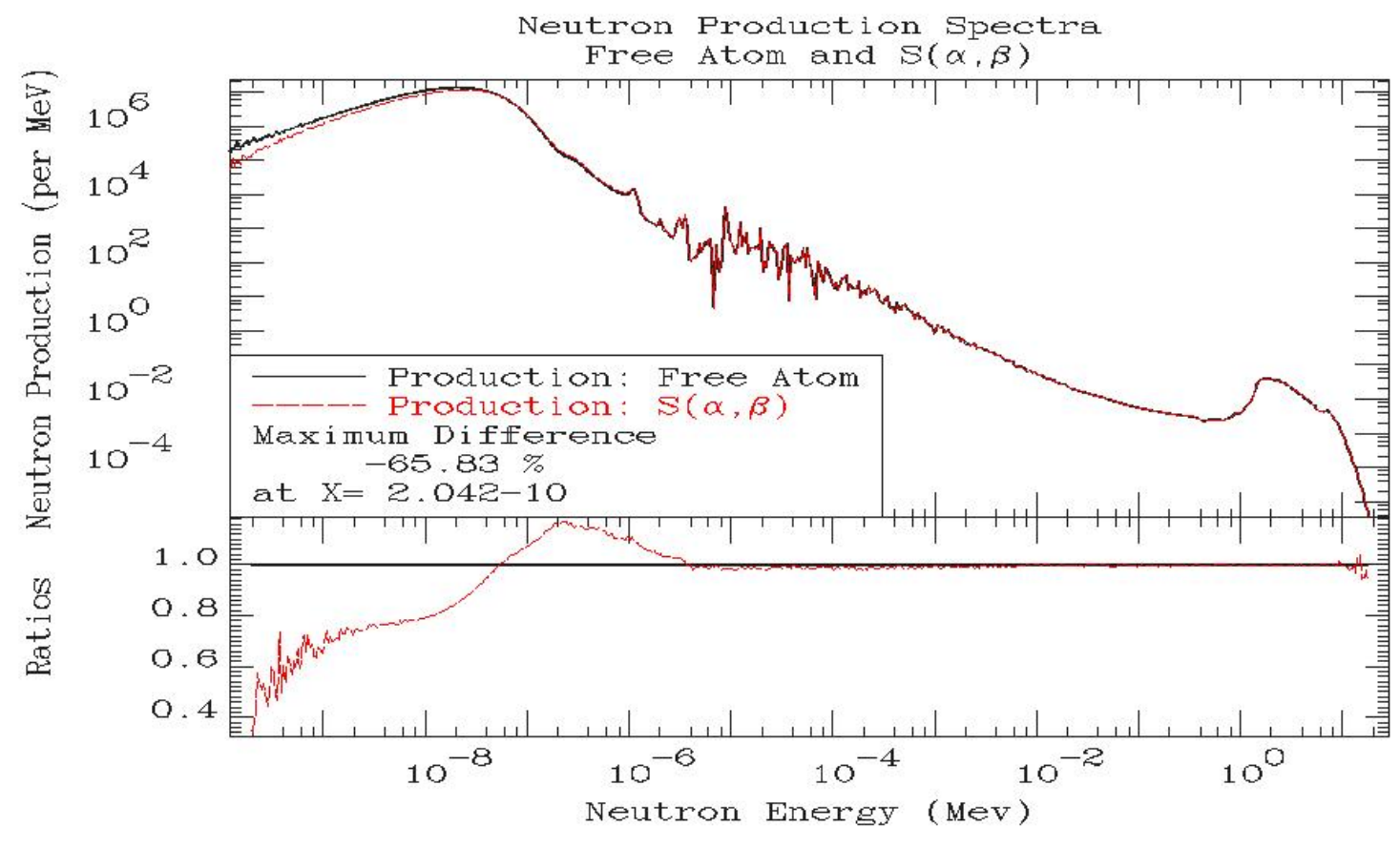

Fig. 4: 1/2" radius fuel pin 


\section{Example \#1: 1/2” Radius Fuel Pin}

Allowing for the difference in K-eff, the important difference between the below two spectra, is that relative to the Free Atom spectrum, the $\mathrm{S}(\alpha, \beta)$ spectrum is shifted toward higher energies in the thermal range, e.g., the $\mathrm{S}(\alpha, \beta)$ results are lower below about $0.06 \mathrm{eV}$ and higher up to above $1 \mathrm{eV}$. This explains the higher median energy of the $\mathrm{S}(\alpha, \beta)$ spectrum, (bound 0.0499 vs. free 0.0452 , over $10 \%$ higher), and lower K-eff (bound 0.961 vs. free 1.011 , roughly $5 \%$ lower), as the spectrum is increased near the low energy uranium capture resonances.

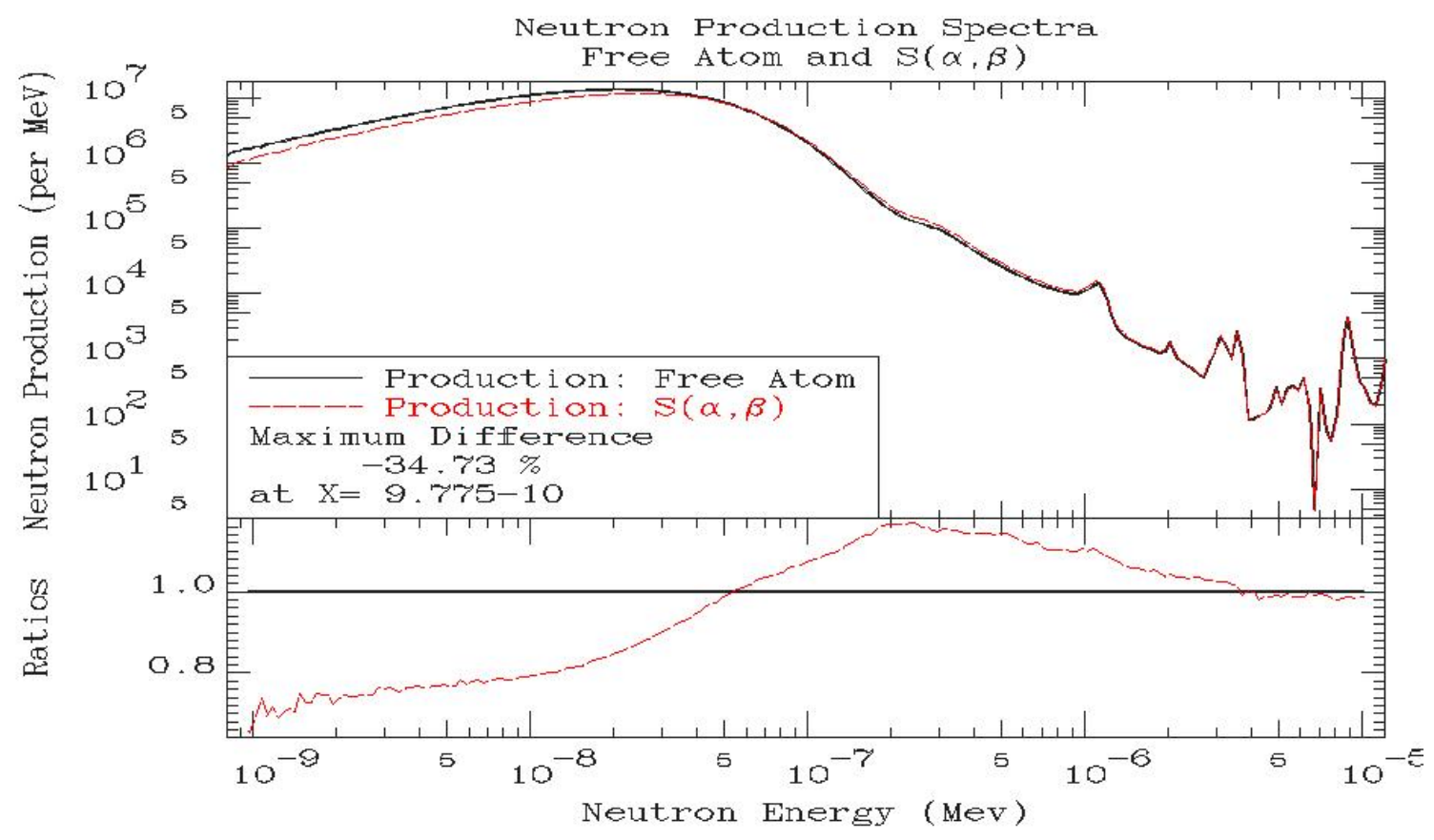

Fig. 5:1/2" radius fuel pin 


\section{Example \#2: 1/4” Radius Fuel Pin}

Allowing for the difference in K-eff, the important difference between the two spectra below, is that relative to the Free Atom spectrum, the $\mathrm{S}(\alpha, \beta)$ spectrum is shifted toward higher energies in the thermal range, e.g., the $\mathrm{S}(\alpha, \beta)$ results are lower below about 0.08 $\mathrm{eV}$ and higher up to above $1 \mathrm{eV}$. This explains the higher median energy of the $\mathrm{S}(\alpha, \beta)$ spectrum (bound 0.0471 vs. free 0.0428 , roughly $10 \%$ higher), and lower K-eff (bound 0.913 vs. free 1.010 , over $9 \%$ lower). Note that the $1 / 4$ " pin spectra are softer (i.e., lower energy) than the above $1 / 2$ " pin results.

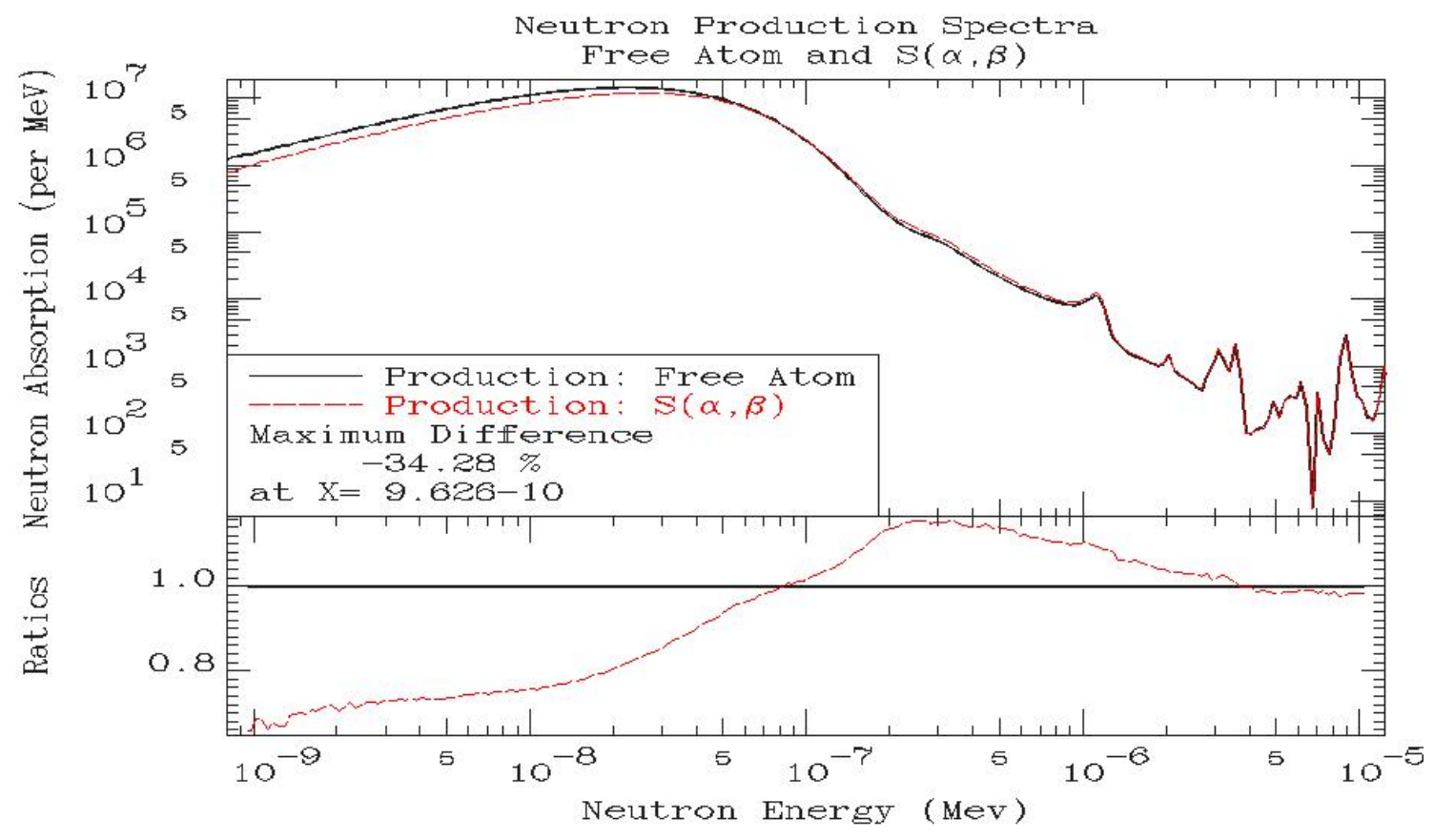

Fig. 6: 1/4" radius fuel pin 


\section{Example \#3: 1/8” Radius Fuel Pin}

Examples \#1 and \#2 involve low enrichment (about 1 and $3 \%$ ). In order to make this system critical would require high enrichment (about $70 \% \mathrm{U}^{235}$ ), which for our thermal reactors is not practical; these results are included only for illustrative purposes. Allowing for the difference in K-eff, the important difference between the below two spectra, is that relative to the Free Atom spectrum, the $\mathrm{S}(\alpha, \beta)$ spectrum is shifted toward higher energies in the thermal range, e.g., the $\mathrm{S}(\alpha, \beta)$ results are lower below about $0.09 \mathrm{eV}$ and higher up to above $1 \mathrm{eV}$. This explains the higher median energy of the $\mathrm{S}(\alpha, \beta)$ spectrum (bound 0.0604 vs. free 0.0546 , roughly $10 \%$ higher), and lower K-eff (bound 0.901 vs. free 1.012 , over $10 \%$ lower). Note that the $1 / 8$ " pin spectra are harder than the above $1 / 2$ " pin results.

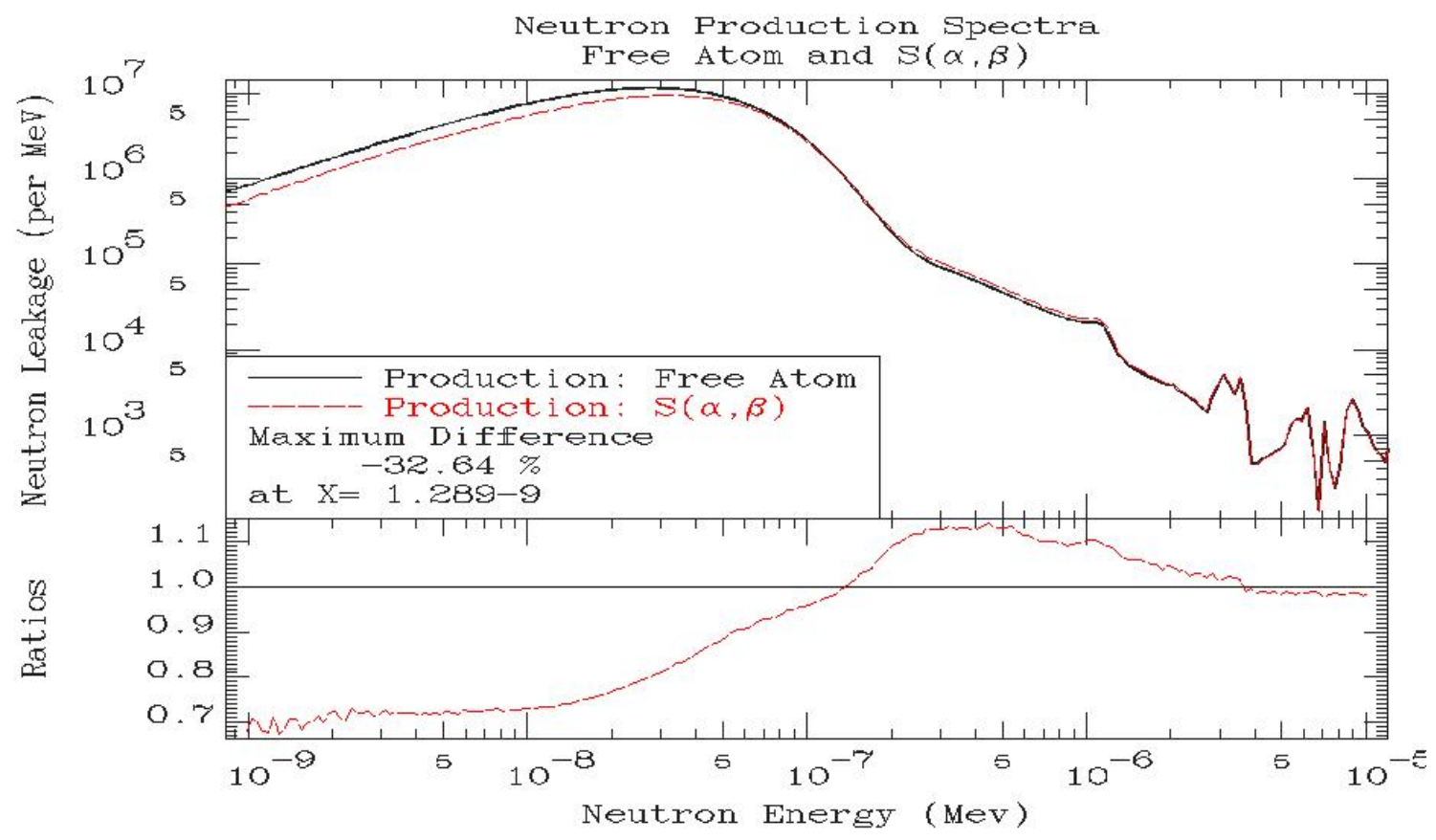

Fig. 7: 1/8" radius fuel pin 


\section{Production and Absorption for the Bound Cases}

Below we show energy dependent production and absorption spectra for the 1/2", 1/4" and $1 / 8$ " bound cases. From these figures we can see which energy ranges are driving the reactivity. For these systems in which there is no leakage, $\mathrm{K}$-eff is defined as,

\section{K-eff $=$ Production/[Absorption + Leakage $]=$ Production/Absorption}

The production and absorption is merely the integral of the energy dependent results shown below. Therefore from these figures we can see the energy range, or ranges, where the ratio of production to absorption is the highest. In all three cases this ratio is small at very low energy because of absorption in the water. In the case of the $1 / 2$ " radius pin this ratio exceeds unity only above about $1 \mathrm{MeV}$. In contrast in the case of the $1 / 4$ " pin, which contains more $\mathrm{U}^{235}$, this ratio also exceeds unity over portions of the resonance region. Finally in the case of the $1 / 8$ " pin, which contains much more $U^{235}$, this ratio exceeds unity over much of the energy range. But remember that even in this case the overall Keff is only about 0.9 , due to heavy absorption in some energy ranges.

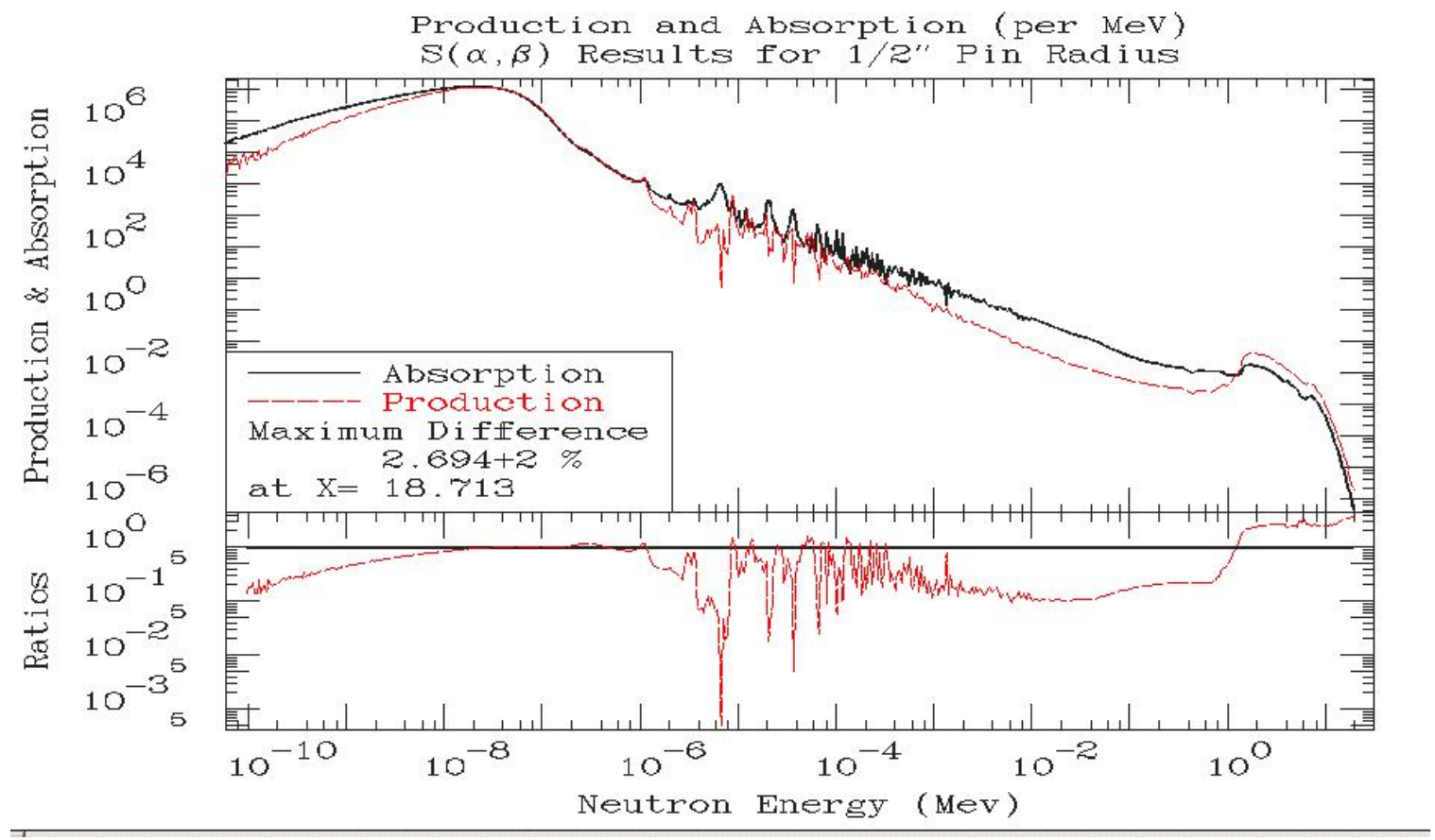

Fig. 8: 1/2" radius fuel pin 


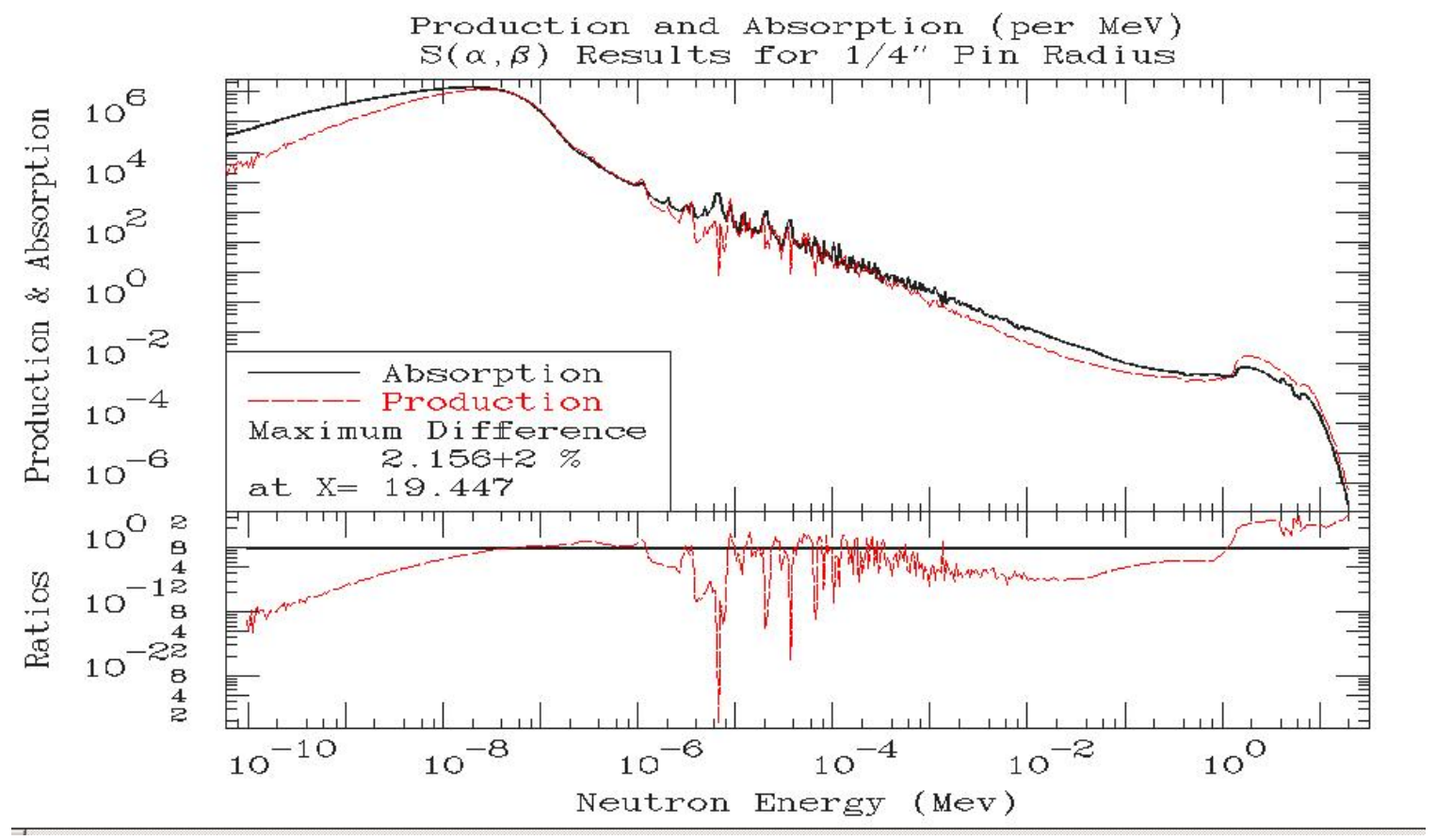

Fig. 9: 1/4" radius fuel pin

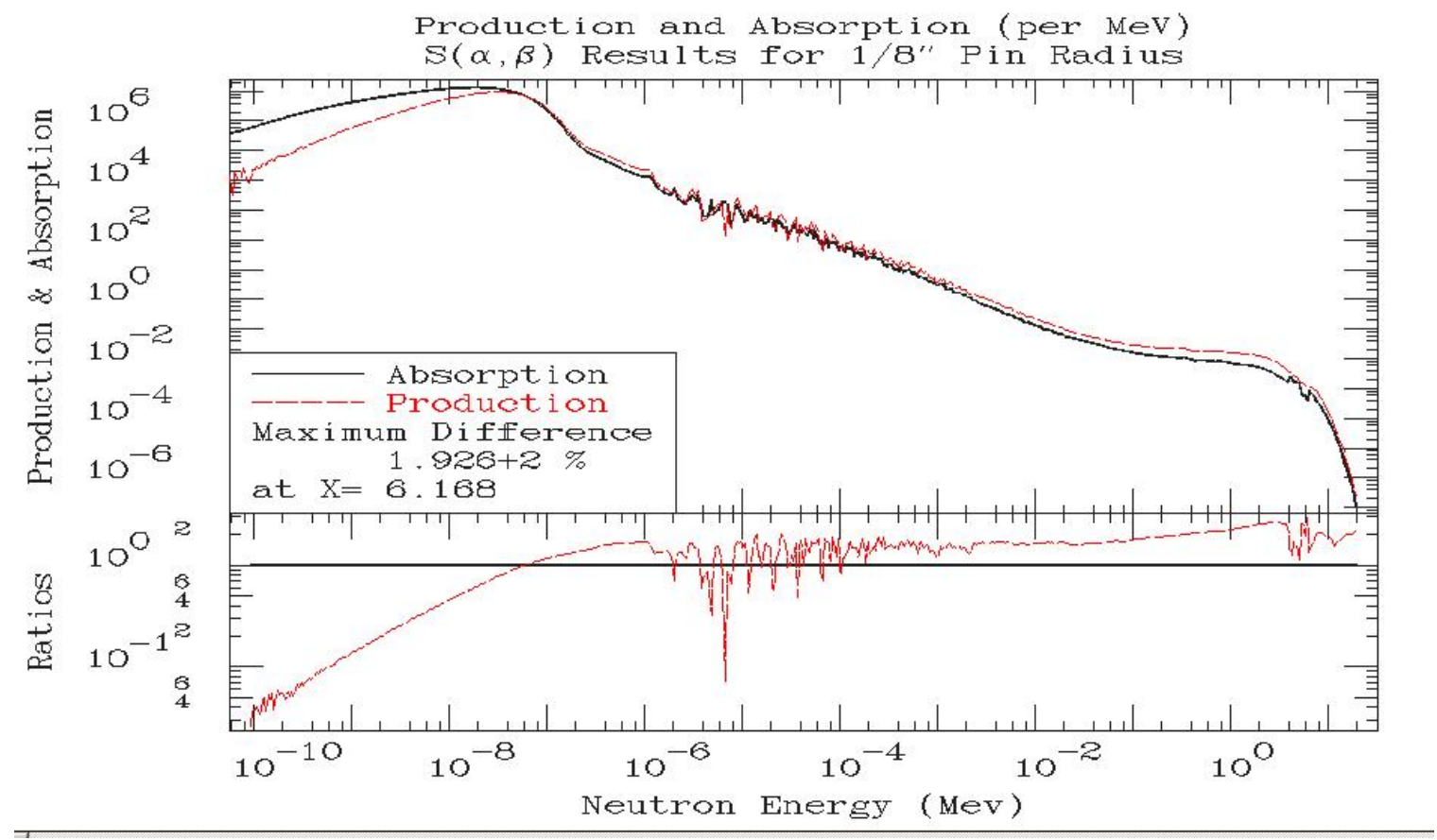

Fig. 10: 1/8" radius fuel pin 


\section{Production Integral for all three bound cases}

By integrating the normalized production spectra we can see a significant difference between the results for $1 / 2 ", 1 / 4$ ", and $1 / 8$ " radius pins. First, in all cases there is little production below about 1 milli-eV, and $80 \%$ to $90 \%$ of the production occurs in a narrow band of energy below $1 \mathrm{eV}$. In the case of the $1 / 2$ " pin which is only $0.98 \% \mathrm{U}^{235}$ and $99.02 \% \mathrm{U}^{238}$, there is a significant contribution due to fast fission in the $\mathrm{U}^{238}$, e.g., almost $10 \%$ of the production is above $1 \mathrm{MeV}$. There is also very little production through the resonance region, e.g., the integral is about the same from $1 \mathrm{eV}$ up to 100 $\mathrm{keV}$. In the case of the $1 / 4$ " pin which is $3.5 \% \mathrm{U}^{235}$ and $96.5 \mathrm{U}^{238}$, there is much less contribution from fast fission, only a few per-cent. There is still very little production through the resonance region. In the case of the $1 / 8$ " pin which is $70 \% \mathrm{U}^{235}$ and $30 \%$ $\mathrm{U}^{238}$, we now see a significant contribution from the resonance region, e.g., between 1 $\mathrm{eV}$ and $100 \mathrm{keV}$ the integral increases by about $10 \%$. See the below plots of the uranium cross sections to understand the differences we see in production.

Note that the median production energy is the energy at which the below integral curve crosses a value of $1 / 2$, e.g., $1 / 2$ the production is below this energy and $1 / 2$ the production is above this energy, which is the definition of the mean value.

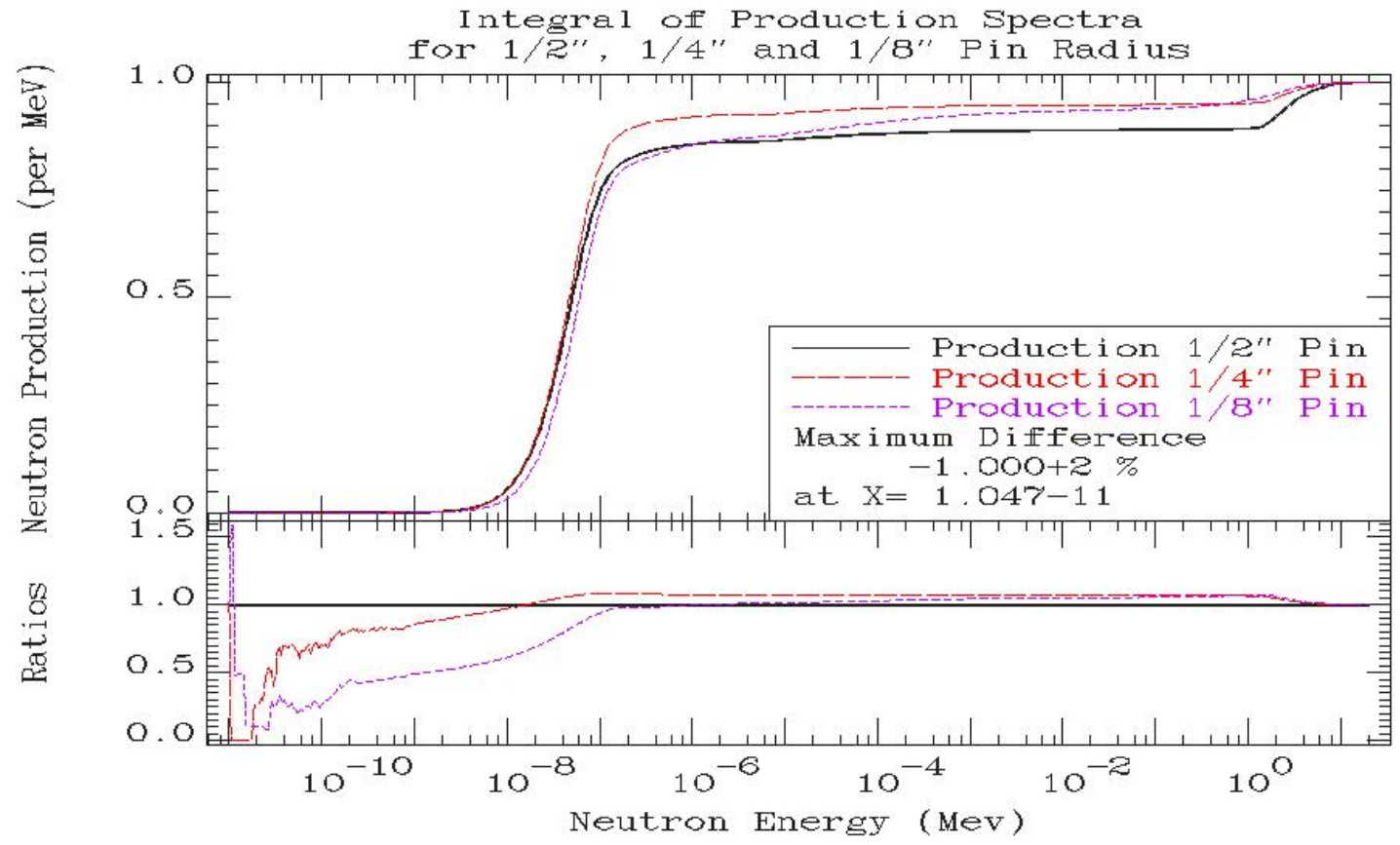

Fig. 11: Integral of Production in 3 cells 


\section{Absorption Integral for all three bound cases}

By integrating the normalized absorption spectra we can see a significant difference between the results for $1 / 2 ", 1 / 4$ ", and $1 / 8$ " radius pins. First, in all cases there is little absorption below about 1 milli-eV, and $80 \%$ to $90 \%$ of the absorption occurs in a narrow band of energy below $1 \mathrm{eV}$. Compared to production we see much less contribution to absorption from fast neutrons. In the case of the $1 / 2$ " pin which is only $0.98 \% \mathrm{U}^{235}$ and $99.02 \% \mathrm{U}^{238}$, there is also significant contribution to absorption through the resonance region, e.g., the integral increases by about $10 \%$ from $1 \mathrm{eV}$ up to $100 \mathrm{keV}$. In the case of the $1 / 4$ " and $1 / 8$ " pins there is almost no contribution from fast fission, and less contribution from the resonance region. See the below plots of the uranium cross sections to understand the differences we see in absorption.

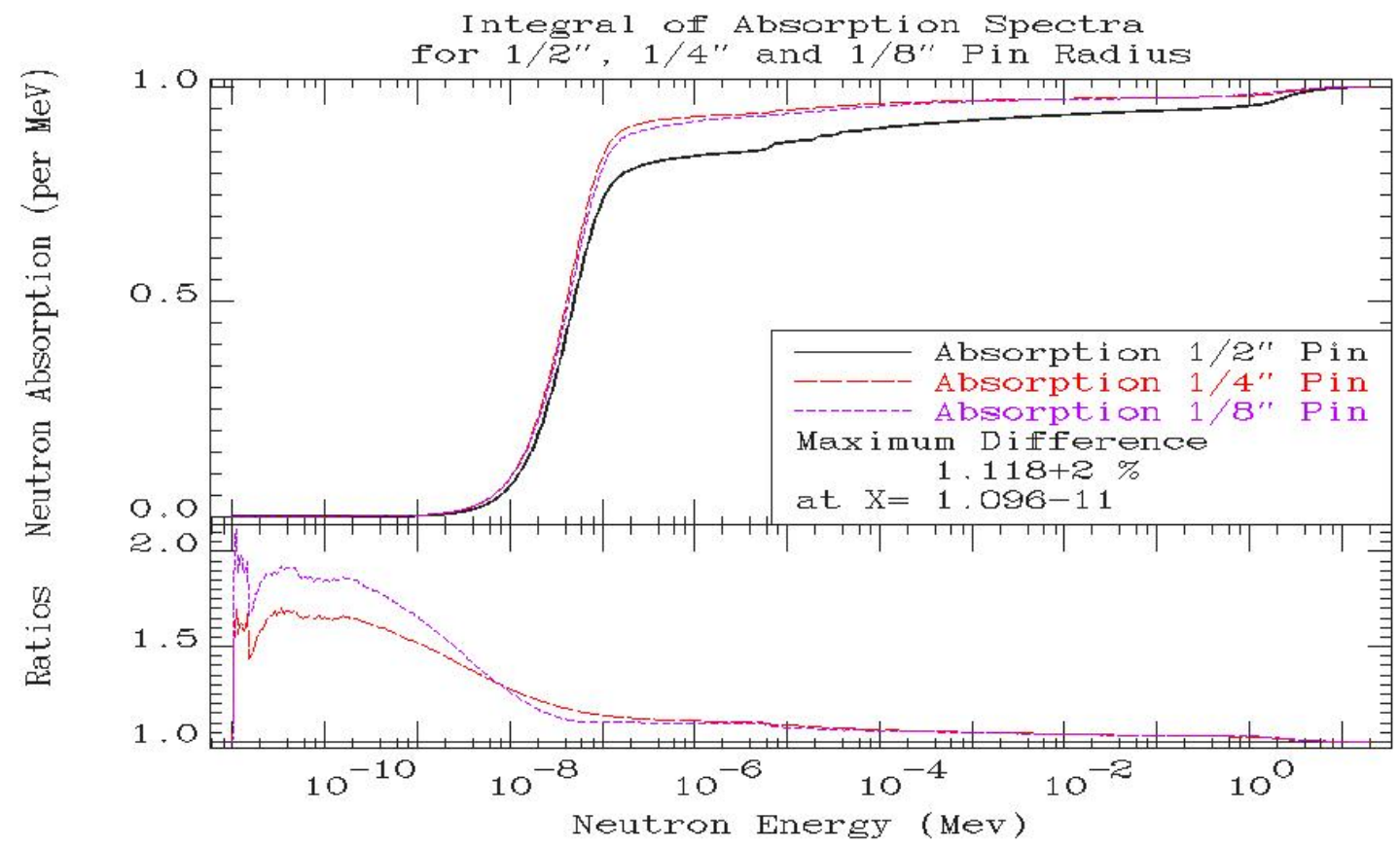

Fig. 12: Integral of Absorption in 3 cells 


\section{Uranium Cross Sections}

From the below plots of the uranium cross sections we can see the decreasing influence of the $\mathrm{U}^{238}$ low energy capture as we go from $1 / 2$ ", $99 \% \mathrm{U}^{238}$, to $1 / 4$ " $96 \% \mathrm{U}^{238}$, to $1 / 8 " 30 \% \mathrm{U}^{238}$. Note in particular the important uranium capture resonances near $1 \mathrm{eV}$.

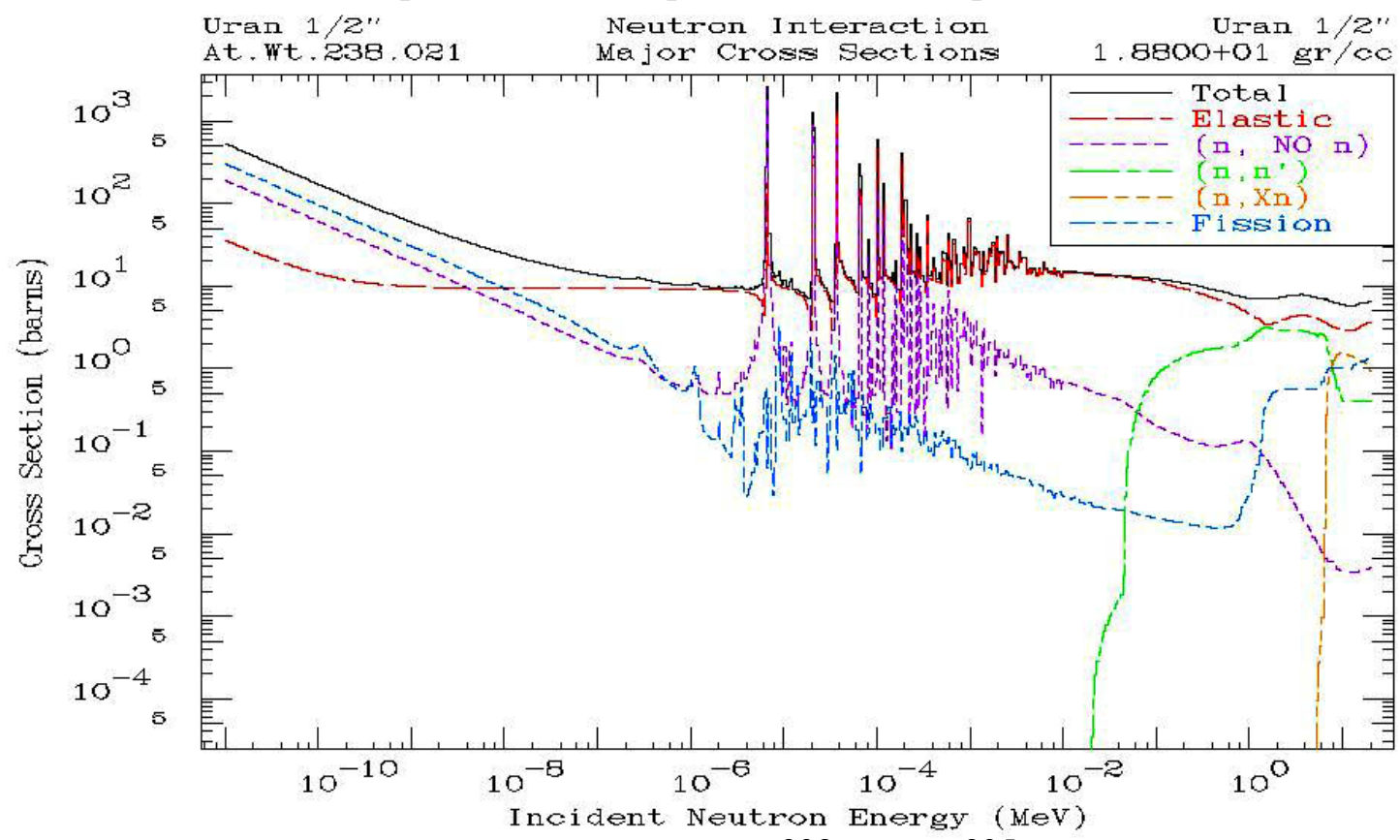

Fig. 13: 1/2", $99.02 \mathrm{U}^{238}, 0.98 \mathrm{U}^{235}$

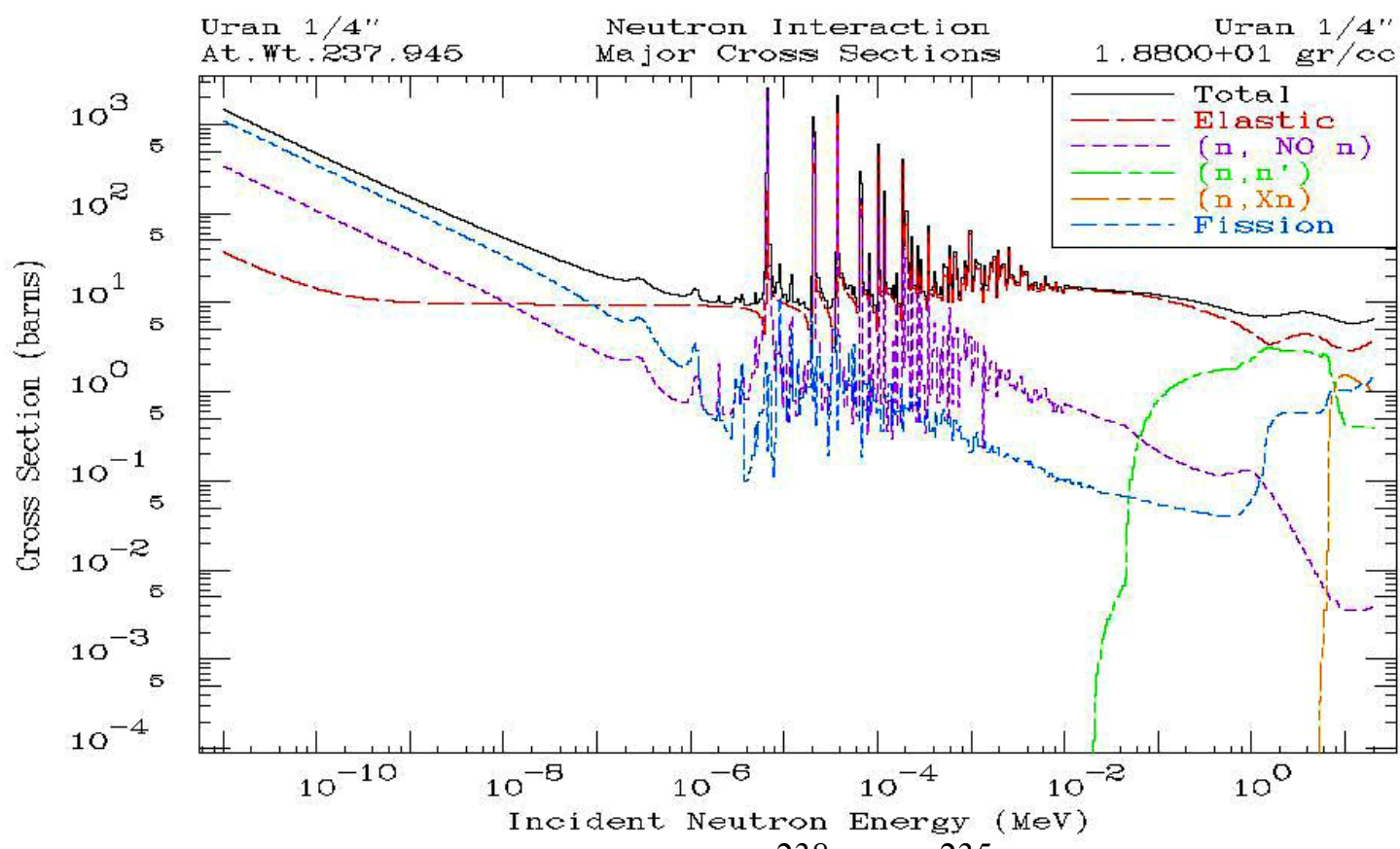

Fig. 14: 1/4", $96.5 \mathrm{U}^{238}, 3.5 \mathrm{U}^{235}$ 


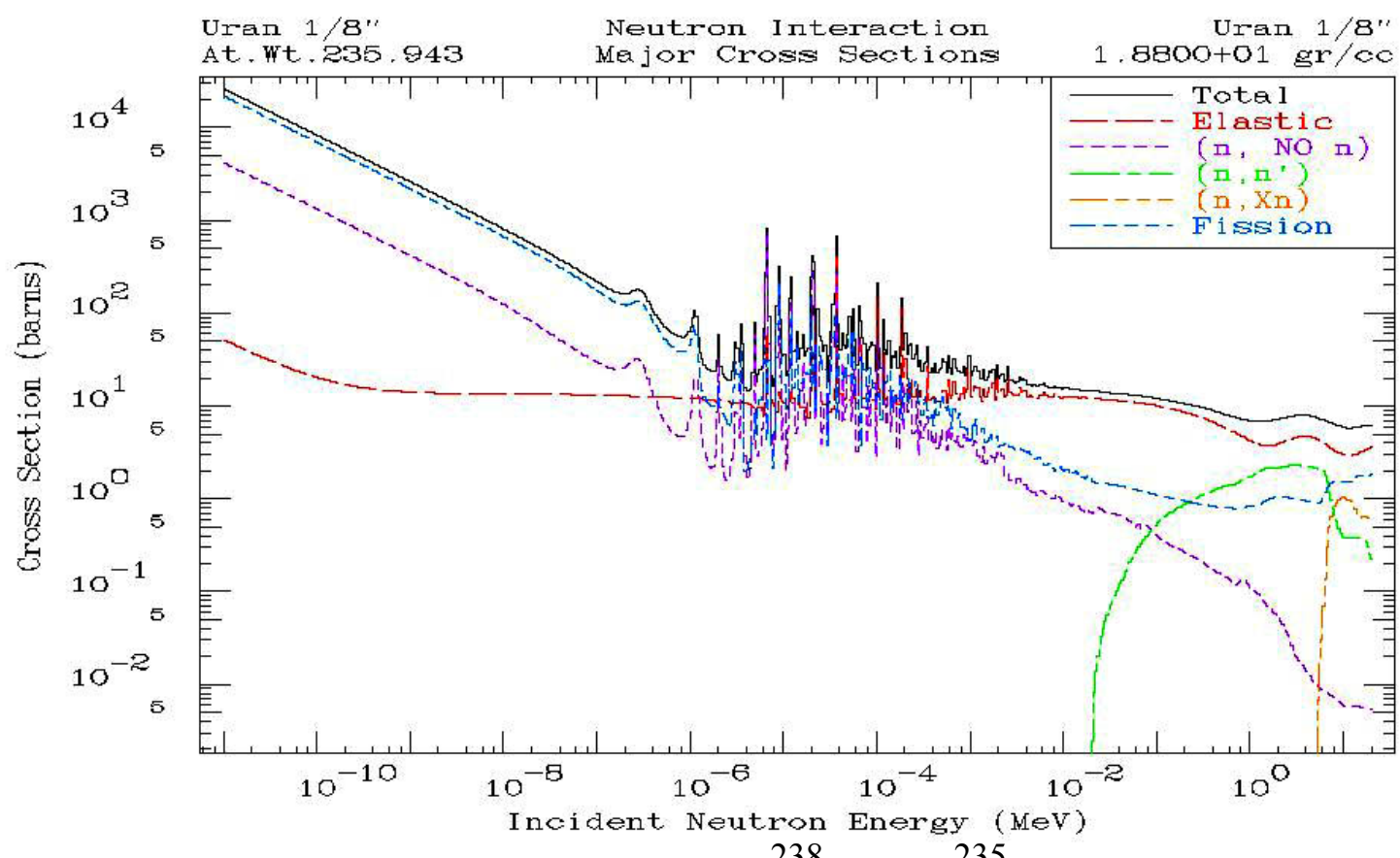

Fig. 15: 1/8", $30.0 \mathrm{U}^{238}, 70.0 \mathrm{U}^{235}$ 


\section{Water Cross Sections}

From the below plots of the Free Atom and Bound hydrogen cross sections for Water, we can see that the bound cross section is considerably higher between roughly 0.001 and 1.0 $\mathrm{eV}$. When using bound cross sections this would tend to decrease the scalar flux in this energy range, i.e., reaction rates tend to remain constant, so that an increase in cross section is usually accompanied by a decrease in flux .

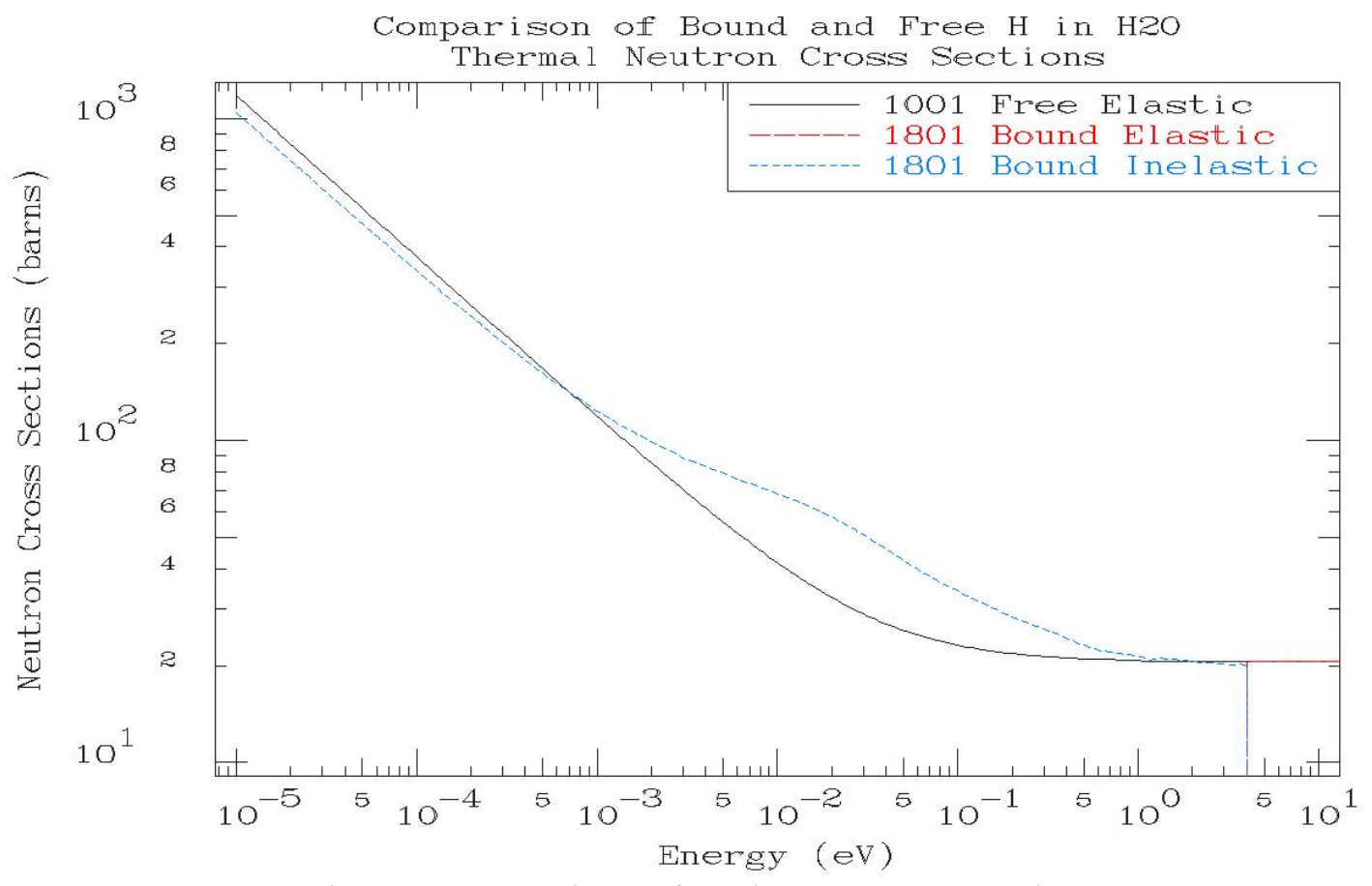

Fig. 16: Comparison of Hydrogen Cross Sections 


\section{General Conclusions}

The important points to learn from these results are,

1) For these systems the effect of the thermal scattering model used is ENORMOUS; the differences in K-eff are at least an order of magnitude greater than the uncertainty of any measured critical assemblies, e.g., for the 1/8" pin K-eff: bound 0.901 vs. free 1.012 .

2) The effect of the thermal scattering model is very system dependent, since we can see that changing the radius of the fuel pin from $1 / 2$ " to $1 / 4$ " changes the effect on K-eff by a factor of roughly two (1/2": $1.0116-0.9614=0.0502$, compared to 1/4": $1.0108-0.9137=0.0971$ )

3) The effect is driven by the heterogeneous nature of the system. I designed these test cases with only uranium in the pin, to minimize slowing down within the pin. Fission neutrons are generated at high energy in the fuel, where the cross sections are low, allowing the neutrons to easily leak from the fuel into the water. In the water they slow down and accumulate at thermal energies, where they then leak back into the fuel. To get accurate results this heterogeneity must be accurately modeled.

\section{Author Specific Conclusions}

Here authors have added their own personal conclusions and remarks.

\section{Red Cullen}

1) Before this study started I did not believe that today Monte Carlo codes had advanced to the state where the K-eff results from many codes would agree to within a small fraction of one per-cent. Based on the results of this study I was wrong, since here we have many Monte Carlo codes in excellent agreement.

2) Before this study started I consulted "experts" and asked many to estimate the effect on K-eff of free versus bound data. All agreed that the difference would be of the order of $1 \%$ or less. Based on the results of this study all of the "experts" were wrong; here we see difference between free and bound results of about 5 to $10 \%$, up to an order of magnitude more than the "experts" predicted.

3) One impetus for this study was to validate our codes so that potentially they could then be used to help in the preparation of "improved" neutron evaluations for future data files, such as ENDF/B-VII. It was hoped that our Monte Carlo code were accurate enough to account for small, about $0.1 \%$, differences in calculated $\mathrm{K}$-eff, that in turn may be able to be justify rather large, $1 \%$ or more, changes in basic cross sections, such as $\mathrm{U}^{238}$ capture. At least to me there is good news and bad news. First the good news is that based on the results of this study our codes 
may be accurate enough to aid in judging such small differences. At least to me the bad news is that the large difference we are seeing due our thermal scattering law data, $10 \%$ in K-eff, may indicate we are looking in the wrong place if we are trying to improve our evaluations. For example, very small changes in our thermal scattering law data result in changes in K-eff by more than the $0.1 \%$ differences we are trying to explain. To me this indicates that it may be time to seriously consider reviewing our thermal scattering law data.

4) At least in my case the results of this study resulted in serendipity, in the sense that based on these results I discovered that I had distributed the wrong neutron data file with TART $2002 \mathrm{CD}$; without this study I might never have discovered, and been able to remedy this problem. In applications the difference in the TART data files only effect thermal systems and results in systems such as these pin cells changing K-eff by about $1 \%$; see details below. TART users who are interested in such systems should contact me to obtain the correct neutron data file.

5) The results of this study have improved not only TART, but also other participating codes, to clarify both preparation of input parameters, and interpretation of output results. This illustrates the importance of periodically comparing code results. Hard work and being conscientious is not enough to guarantee the accuracy and reliability of our codes. None of our codes are perfect and the easiest way I know of to find and fix flaws in our codes is by comparison studies, such as this one.

\section{Roger Blomquist}

1) Although the inter-comparisons with thermal scattering are useful, the free-atom results are not of themselves very interesting, except that they demonstrate the importance of using thermal scattering to correctly model these systems. It is possible that the free-atom comparisons might point to problems in a code or library, but it is very unlikely that these will be important in real physical systems.

\section{Christopher Dean}

1) This benchmark is designed to test thermal scattering data. The results emphasize this effect beyond the level seen in practical thermal reactors where less water is present around pins. This may explain why the difference between use of free and bound scattering is larger than "the experts" initially envisaged.

2) MONK results from Winfrith apply our released code MONK8b. The results for the $1 / 8$ " case used 10,000 neutrons per stage (cycle) to obtain the production spectrum requested. When the same sampling was applied to the $1 / 4$ " and $1 / 2$ " cases statistical noise remained in the spectrum. These two calculations were then repeated with 100,000 neutrons/stage for some $\sim 600$ stages to remove the noise. These latter cases take many days to run on the available PC machines. 
3) A new version of MONK is being developed with a continuous nuclear data representation. The benchmark has been applied to this MONK9 code with its BINGO library. For interest we have included results but as yet only using 10,000 neutrons per stage. Already the differences seen are larger than were expected and will be investigated before the code is issued.

\section{Dave Heinrichs}

1) This study demonstrates the importance of molecular binding effects in thermal neutron scattering and its large impact on k-eff in some systems. I wonder how much of the calculated discrepancy seen in critical UF6-HF systems published in the ICSBEP Handbook may be due to these effects.

2) I was also very surprised at the differences in k-eff definitions used in various codes; namely, definitions based on "fission generations" versus "reaction rates" and their possible impact on calculated spectra. These issues warrant further study.

\section{Yi-Kang Lee}

1) Before the round zero started I asked Dr. Cullen to precise some basic specifications for this benchmark. My questions included the temperatures of the moderator (water) and the fuel, the evaluation/version of nuclear data used by his code, the composition of fuel and water in the way ' $\mathrm{n} \times 1 \mathrm{E}-24$ atoms. $\mathrm{cm}^{-3}$, , the definition and the tally locations (fuel or fuel cell) of the 'median fission energy' and the 'neutron production spectra', the impact of the tally bins number (172 instead of 641), etc. Dr. Cullen only replied me that this is room temperature and theoretical K-eff problem. I understood that we compare not only the Monte Carlo code and the thermal scattering treatment but also the data library and the user's experience.

2) TRIPOLI-4 code can run with nuclear data of different temperature and of different evaluations with ENDF/B-VI format (JEF2.2, JEF3.0, ENDF/B-VI.4, ENDF/B-VI.8, JENDL3.2, JENDL3.3, etc.). My previous experience on similar benchmarks, PWR critical lattice (LCT-008) and MARACAS critical configurations (LCT-049), showed that only using JEF2.2 and ENDF/B-VI could produce a difference about $0.5 \%$ (LCT-008) and $0.8 \%$ (LCT-049) in K-eff calculation. To reduce the impact of the data library comparing with Dr. Cullen's TART code, continuous-energy nuclear data of $300 \mathrm{~K}$ from ENDF/B-VI.4 were used in present TRIPOLI-4 calculations.

3) In fact, except nuclear data evaluation (JEF, ENDF/B, JENDL and other evaluations), cross-section processing method (NJOY94.xx, NJOY99.xx and other processing tools), thermal scattering treatment tool (THERMR-91, THERMR-99 and other tools) and thermal scattering in different temperature (300 $\mathrm{K}$ or other temperatures) would change the Monte Carlo K-eff result. For Monte 
Carlo codes used in this study, it is interesting and necessary to compare these items so as to understand how to make difference of results.

4) This study started when I was preparing two papers for the coming conference ICRS-10 with TRIPOLI-4 code. The first paper evaluates the albedo-type TLD personal dosimeter neutron response (neutron thermal scattering in water-filled ISO phantom) and the second paper investigates the N50 neutron slab monitor detection efficiency (neutron thermal scattering in polyethylene moderator). In present study, the effect on K-eff of free versus bound data was taken into account and in those two papers, the effects on albedo-type TLD response and on neutron monitor detection efficiency of free versus bound data were considered. With free atom model, the CPU running time of TRIPOLI-4 can be largely reduced but overestimation in TLD dosimeter response (18\% in $1 \mathrm{MeV}$ neutron source case) and in N50 neutron monitor detection efficiency (30\% in heavily moderated case) can also be obtained.

\section{Robert MacFarlane}

1) Unfortunately, all MCNP users do not currently have easy access to MCNP5 and the new libraries that come with it. The new ENDF66 library adds materials processed from Release 6 (.66c ZAIDs). Additional Release 8 materials are in the ACTI library with .62c ZAIDs. Oxygen is the interesting one here. The new SAB2002 library of thermal scattering data is based on the Release 3 evaluations of $S(\alpha, \beta)$, uses more discrete energies and angles, and was processed with tighter convergence $(.60 \mathrm{t}, .61 \mathrm{t}, \ldots)$. The following sensitivities may help people to compare different MCNP results: R8 (.66c+8016.62c+.60t) .96062(13), same with UR turned off .95996(13), same except .01t .96036(13), R6 (.66c+.60t) $.96030(12)$, R2 (.60c $+.01 \mathrm{t}) .95878$. Note that the change in the continuous energy cross sections from R2 (ENDF60) to R8 increases $k$, while the change in the thermal data decreases $\mathrm{k}$. As a result, MCNP5 with the new libraries gives very close to the same answer as MCNP4 with the old libraries, but the agreement doesn't say anything about how good the thermal data are. Until all the calculations are made with equivalent data, one must be careful in drawing conclusions from the table in this report about the accuracy of the thermal methods.

\section{Yasunobu Nagaya}

1) The results have made us realize again the importance of thermal scattering data. It is therefore desired that the data are also continuously improved as well as cross section data.

2) For code-to-code comparison, we can see the spread of the results by $\sim 1 \%$. The dominant cause would be mostly due to the difference of used nuclear data. But the difference of thermal scattering treatment in Monte Carlo codes would be 
another cause. We believe that the difference effects the K-eff values by less than $\sim 0.2 \%$. Quantitative investigation will be required in the future.

\section{Andrej Trkov}

1) This benchmark exercise was a good opportunity to verify the accuracy and reliability of deterministic lattice codes such as WIMS-D and the data processing methods from the WIMS-D Library Update Project of the IAEA. The results are favorable, especially with the 172-group library. Indirectly this benchmark also validates the methods for generating the multi-group scattering cross section matrices in the thermal energy range from tabulated $S(a, b)$ data. In the present set of test cases with large pin pitch the approximation of a square cell and reflective boundary conditions with an equivalent cylindrical cell and white boundary conditions does not seem to be very important.

2) Verification was performed to check that different versions of MCNP (on different machines) actually produce the same result (within the statistical uncertainty) when the same data are used. This verification was done with data libraries of the ".60c" series with thermal scattering law file "lwtr.01t", which are distributed with packages MCNP-4C and MCNPX-2.4.0. The results are shown in runs $10-12$. Run 14 was done with a library generated locally with NJOY from ENDF/B-VI, R8 data and is comparable to run 8 by Red Cullen. The comparison verifies that the results are reproducible. Runs 13 shows the difference when the old "lwtr.01t" scattering law data are used. Run 15 tests the sensitivity on the new scattering law data and are discussed below.

3) As a result of an IAEA project, $M$. Mattes has recalculated the thermal scattering law data $S(\alpha, \beta)$ for hydrogen bound in water. The new data are tabulated on a denser mesh of $\alpha, \beta$ and temperature for energy transfer ranges comparable to the ones in the ENDF/B-VI library. Thermal scattering law data affect the predicted reactivity, but the results of the present analysis are inconclusive. Deterministic calculations suggest an increase of reactivity (run 32 vs. 31), particularly for the 1/8" case, while Monte Carlo calculations indicate a slight decrease (run 15 vs. 14). The differences due to different scattering law data with reference to ENDF/B-VI are summarized in Table 3. The same methods were used to process the $S(\alpha, \beta)$ data from different sources. Interpretation of the results is obscured by the observation that Monte Carlo results from different codes for the "free" $1 / 8$ " case using ENDF/B-VI, R8 data (for example, run 8 and 24) differ by about 90 pcm, while the "bound" results are discrepant by more than $400 \mathrm{pcm}$. If the difference in the "free" results is systematic, the difference due to $S(\alpha, \beta)$ for the bound case becomes even larger. Such large differences in the results from codes that use the same source nuclear data need to be resolved before any conclusion can be drawn about the impact of the new scattering law data.

Table 3: Difference due to S(a,b) data in MCNPX-2.4.0 and WIMS-D results. 


\begin{tabular}{|c|c|c|l|}
\hline $1 / 2 "$ case & $1 / 4^{\prime \prime}$ case & $1 / 8$ " case & Comment \\
\hline-11 & -40 & -24 & MCNPX-2.4.0 \\
17 & 87 & 217 & WIMSD-5B \\
\hline
\end{tabular}

\section{References}

[1] COG - A Multiparticle Monte Carlo Transport Code, User's Manual, Fifth Edition, Richard M. Buck, Edward Lent, Tom Wilcox, Stella Hadjimarkos, UCRL-TM-202590, September 1, 2002.

[2] KENO V.a: An Improved Monte Carlo Criticality Program with Supergrouping, L.M. Petrie and N.F. Landers, NUREG/CR-0200, Revision 6, Volume 2, Section F11, ORNL/NUREG/CSD-2/R6, September 1998.

[3] MCNP - A General Monte Carlo N-Particle Transport Code, Version 5, Volume I: Overview and Theory, X-5 Monte Carlo Team, Los Alamos National Laboratory report LA-UR-03-1987 (April 24, 2003). Portions of the MCNP manual are available on-line at http://www-xdiv.lanl.gov/x5/MCNP/themanual.html

[4] The MCU/REA code with Data Library DLC $\backslash$ MCUDAT-2.2. (In Russian). Voprosy atomnoy nauki and Techniki. Ser. FYaR. Issue. 3, c. 55-62, 2001.

[5] The Answers Software Package MONK. A Monte Carlo Program for Nuclear Criticality Safety Analysis. User guide for Version 8. Updates for version 8B June 2001 ANSWERS/MONK(98)6, Serco Assurance, A32, Winfrith, Dorchester, Dorset, DT2 8DH, UK

[6] MVP/GMVP: General Purpose Monte Carlo Codes for Neutron and Photon Transport Calculations based on Continuous Energy and Multigroup Methods, JAERIData/Code 94-007, July 1994 by Takamasa Mori and Masayuki Nakagawa [in Japanese]. The English manual for MVP 2.0 is to be published this year.

[7] TART 2002: A Coupled Neutron-Photon 3-D, Combinatorial Geometry, Time Dependent Monte Carlo Transport Code, Report: UCRL-ID-126455, Rev. 4, Code Release: UCRL-CODE-2003-043, by D.E. Cullen, July 2003, Lawrence Livermore National Laboratory. TART 2004, to be published. TART 2002 report is available on-line at http://www.llnl.gov/cullen $1 / \mathrm{mc} . h \mathrm{tm}$

[8] TRIPOLI-4.3 User Manual for version 4.3 of the TRIPOLI-4 Monte Carlo method particle transport computer code, CEA-R-6044, by J. P. Both, A. Mazzolo, O. Petit, Y. Peneliau, B. Roesslinger, CEA/Saclay, France, November 2003. TRIPOLI-4 version 4.3.2 is available from $\underline{\mathrm{http}} / / / \mathrm{www} . n$ ea.fr/abs/html/nea-1716.html

[9] Status of the VIM Monte Carlo Neutron/Photon Transport Code, by R. N. Blomquist, Proceedings of the $12^{\text {th }}$ Biennial RPSD Topical Meeting, Santa Fe, NM, April 14-18, 2002. Separate VIM User's Manual available with the code from RSICC. 
[10] WIMS: ASKEW, J. R., FAYERS, F. J., KEMSHELL, P. B., A General Description of the Code WIMS, J. British Nucl. Energy Soc., Vol. 5, No. 4, p.564, October 1966, WIMS-D5, NEA Data Bank Documentation, Package ID No. 1507/02, 1998.

[11] WIMS-ANL 4.0 Deterministic Code System for Lattice Calculation, J.R. Deen, W.F. Woodruff, C.I. Costescu and L.S. Leopando, Argonne National Laboratory, RSICC Code Package CCC-698, 2000. 


\section{APPENDICES}

\section{Contact Information}

This section includes the complete addresses of all participants, for your use should you wish to contact them,

\section{Dermott E. Cullen}

University of California

Lawrence Livermore National Laboratory

P.O.Box 808/L-159

Livermore, CA 94550

Tele: $925-423-7359$

E.Mail: cullen1@1lnl.gov

Website: http://www.llnl.gov/cullen1

\section{Roger N. Blomquist}

Argonne National Laboratory (208-E111)

9700 S. Cass Ave.

Argonne, IL 60439

Tele: 630-252-8423

E Mail: RNBlomquist@anl.gov

\section{Christopher John Dean}

Room 347 Building A32,

Winfrith Technology Centre,

Winfrith,

Dorchester,

Dorset,

DT2 8DH

United Kingdom.

Tele: (UK) $\quad 01305851150$

Tele:(International) 441305851150

E-mail christopher.dean@,sercoassurance.com

\section{Dave Heinrichs}

University of California

Lawrence Livermore National Laboratory

P.O. Box 808, L-198

Livermore, CA 94551-0808

Tel: 925-424-5679

E.Mail: heinrichs1@,llnl.gov

\section{Mikhail A. Kalugin}

RRC "Kurchatov Institute"

Kurchatov Sq. 1, 
123182 Moscow,

Russian Federation.

E.Mail: Kalugin@adis.vver.kiae.ru

Mark Lee, DOE/LSO

P.O.Box 808/L-293

Livermore, CA 94550

Tele: 924-424-4567

E.Mail: Mark.Lee@oak.doe.gov

Yi-Kang Lee

CEA/Saclay,

DEN/DM2S/SERMA/LEPP, Bat. 470,

91191 Gif sur Yvette,

France

Tele: 33169088479

Fax: 33169084572

E.Mail: $\underline{\text { YKLee@cea.fr }}$

Robert MacFarlane

MS B243 T-16

Los Alamos National Laboratory

Los Alamos, NM 87545 USA

Tele: 505-667-7742

E.Mail: ryxm@lanl.gov

Yasunobu Nagaya

Japan Atomic Energy Research Institute

Tokai-mura, Naka-gun, Ibaraki-ken, Japan 319-1195

Tele: +81-29-287-3064

E.Mail: Nagaya@mike.tokai.jaeri.go.jp

Andrej Trkov,

International Atomic Energy Agency

Wagramerstrasse 5

P.O.Box 100

A-1400 Vienna

Austria

Tele.: +431260021712

E.Mail: $\underline{\text { A.Trkov@iaea.org }}$

\section{Explanation of Round Zero Errors}

Here each author can add their own personal explanation of initial errors found during the Round Zero comparisons. 
TART - Red Cullen: There was serendipity from this study, in the sense that even though we were not looking for this affect I discovered that I had erroneously distributed an experimental data file with TART $2002 \mathrm{CD}$ which led to my Round Zero results that were about $1 \%$ too low for K-eff of these cells. The below table presents the Round Zero results using the incorrect data file with tart02, and the correct data file using tart04. This only effects thermal systems. Without participating in this study it is unlikely that this error would have been found. Once found it was simple for me to make the correct data file available to Tart users.

\begin{tabular}{|c|c|c|c|c|c|c|}
\hline K-eff & $1 / 2^{\prime \prime}$ & & $1 / 4^{\prime \prime}$ & & $1 / 8^{\prime \prime}$ & \\
\hline & Free & bound & free & bound & free & bound \\
\hline TART02(Red) & $1.00080(5)$ & $0.95214(5)$ & $0.99938(5)$ & ๑.90588(5) & $1.00099(5)$ & $0.89507(5)$ \\
\hline TART04(Red) & $1.00952(5)$ & $0.96061(5)$ & $1.00930(5)$ & $\odot .91539(5)$ & $1.01120(5)$ & $0.90304(5)$ \\
\hline
\end{tabular}

TART - Mark Lee: Beginner users of a code have to be wary not only of making mistakes with input, but in their interpretation of the output. For my first set of TART runs I misinterpreted the output file and reported the wrong set of K-eff values (the average K-eff reported at the end of the output file.) Dr. Cullen has now modified TART to highlight the recommended value of K-eff for future users.

WIMSD-5B - Andrej Trkov: The generic WIMS libraries have the resonance integrals for $\mathrm{U}^{235}$ tabulated as a function of the dilution cross section $\operatorname{Sig} 0$ down to 200 barns. This is sufficient for most critical lattices, but the $1 / 8$ " case with relatively high $\mathrm{U}^{235}$ requires a much lower Sig0 value. The resonance tables were extrapolated in the WIMS$\mathrm{D}$ code without a warning. For the purpose of this exercise the data for $\mathrm{U}^{235}$ in the 172 group library were processed again with resonance integrals tabulated down to Sig0 of 50 barns. Both sets of results are included.

Red Cullen - I will again mention that over $30 \%$ of the initial, round zero results were in error. I am sorry to see that other authors did not take this opportunity to admit that they and their code and data bases are not perfect, and to try to help their code users by describing potential pitfalls in using their codes. We all know the codes are not perfect, and yet it is very different to get people to publicly admit it. So let me try to describe the generic types of problems we found during round zero, without my mentioning specific codes. First of all we did not find anything basically wrong with any of the codes or their data bases. What we did find was the weakest link is the code user. All of the incorrect results we found during round zero were due to misuse of the codes by users, in the sense that they used the wrong input options, and in one case misinterpreting the code results in reporting the wrong K-eff values. This may seem trivial, but it is not; please appreciate that if it were not for the comparisons we were performing the code users would have believed their erroneous results, and continued misusing the codes. This indicates to me that the codes need more human engineering, in an attempt to make them more user friendly. It also highlights the importance of comparing code results. I routinely check important results by comparing TART, MCNP and COG results. These days we have an abundance of readily available, cheap computer power, and I try to use it to verify my results.

\section{Submitting Code Results}


This is an ongoing study that will not end with the publication of this report. Therefore if you, a reader, would like to submit results please feel free to do so; and your results will be included in the next published version of this report.

In order to minimize the amount of work done by everyone, I ask you to submit results ONLY for EXACTLY the systems defined above. Please do not try to be creative and change the systems in any way. If you submit results for any other systems I will not be able to use them, and you will end up merely wasting your time.

As a minimum you are asked to provide K-eff, and if available the median fission energy; not the average, the median. The median fission energy can be calculated from the neutron production spectrum, as shown above.

If available the neutron production, absorption and leakage spectra would be very useful in attempting to explain any differences that we see between code results. Most convenient would be if you provide the spectra using 50 tally bins per energy decade, with the bins equally spaced in the log of energy between, $10^{-5} \mathrm{eV}$ and $20 \mathrm{MeV}$ (616 bins). For the convenience of MCNP users, below I include these 616 energies that can be used as a part of an MCNP input deck.

\footnotetext{
e1 $\quad 1.00000 \mathrm{E}-11$ 1.04713E-11 $1.09648 \mathrm{E}-11$ 1.14815E-11 $1.20226 \mathrm{E}-11$ 1.25893E-11 1.31826E-11 1.38038E-11 1.44544E-11 1.51356E-11 1.58489E-11 1.65959E-11 1.73780E-11 1.81970E-11 1.90546E-11 $\begin{array}{lllll}1.99526 \mathrm{E}-11 & 2.08930 \mathrm{E}-11 & 2.18776 \mathrm{E}-11 & 2.29087 \mathrm{E}-11 & 2.39883 \mathrm{E}-11\end{array}$ 2.51189E-11 2.63027E-11 2.75423E-11 2.88403E-11 3.01995E-11 3.16228E-11 3.31131E-11 3.46737E-11 3.63078E-11 3.80189E-11 3.98107E-11 4.16869E-11 4.36516E-11 4.57088E-11 4.78630E-11 5.01187E-11 5.24807E-11 5.49541E-11 5.75440E-11 6.02560E-11 6.30957E-11 6.60693E-11 6.91831E-11 7.24436E-11 7.58578E-11 7.94328E-11 8.31764E-11 8.70964E-11 9.12011E-11 9.54993E-11 $1.00000 \mathrm{E}-10$ 1.04713E-10 1.09648E-10 1.14815E-10 $1.20226 \mathrm{E}-10$ $1.25893 \mathrm{E}-10$ 1.31826E-10 1.38038E-10 $1.44544 \mathrm{E}-101.51356 \mathrm{E}-10$ $1.58489 \mathrm{E}-10$ 1.65959E-10 1.73780E-10 $1.81970 \mathrm{E}-101.90546 \mathrm{E}-10$ $\begin{array}{lllll}1.99526 \mathrm{E}-10 & 2.08930 \mathrm{E}-10 & 2.18776 \mathrm{E}-10 & 2.29087 \mathrm{E}-10 & 2.39883 \mathrm{E}-10\end{array}$ $2.51189 \mathrm{E}-10 \quad 2.63027 \mathrm{E}-10 \quad 2.75423 \mathrm{E}-10 \quad 2.88403 \mathrm{E}-10 \quad 3.01995 \mathrm{E}-10$ $3.16228 \mathrm{E}-10 \quad 3.31131 \mathrm{E}-10 \quad 3.46737 \mathrm{E}-10 \quad 3.63078 \mathrm{E}-10 \quad 3.80189 \mathrm{E}-10$ 3.98107E-10 4.16869E-10 4.36516E-10 4.57088E-10 4.78630E-10 5. $01187 \mathrm{E}-10 \quad 5.24807 \mathrm{E}-10 \quad 5.49541 \mathrm{E}-10 \quad 5.75440 \mathrm{E}-10 \quad 6.02560 \mathrm{E}-10$ $6.30957 \mathrm{E}-10 \quad 6.60693 \mathrm{E}-10 \quad 6.91831 \mathrm{E}-10 \quad 7.24436 \mathrm{E}-10 \quad 7.58578 \mathrm{E}-10$ 7.94328E-10 8.31764E-10 8.70964E-10 9.12011E-10 9.54993E-10 1. $00000 \mathrm{E}-09$ 1.04713E-09 1.09648E-09 1.14815E-09 1.20226E-09 1.25893E-๑9 1.31826E-@9 1.38038E- $\odot 9$ 1.44544E- 09 1.51356E- 09

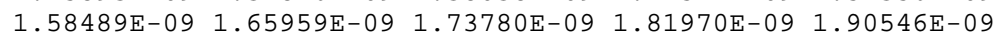

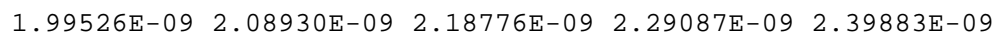
2.51189E-๑9 2.63027E-๑9 2.75423E- $\odot 9$ 2.88403E- $\odot 9$ 3. $01995 \mathrm{E}-\odot 9$ 3.16228E-๑9 3.31131E-@9 3.46737E- 09 3.63078E- 09 3.80189E- 09 3.98107E-๑9 4.16869E-@9 4.36516E- 09 4.57088E- 09 4.78630E- 09 5.01187E-09 5.24807E-09 5.49541E-09 5.75440E-09 6.02560E-09 6.30957E-๑9 6.60693E-09 6.91831E- 09 7.24436E- 09 7.58578E-๑9 7.94328E-09 8.31764E-09 8.70964E-09 9.12011E-09 9.54993E-09 1.00000E-08 1.04713E-08 1.09648E-08 1.14815E-08 1.20226E-08 1.25893E-๑8 1.31826E-08 1.38038E- 08 1.44544E- 08 1.51356E- 08 1.58489E-08 1.65959E-08 1.73780E-08 1.81970E-08 1.90546E-08 $\begin{array}{lllll}1.99526 \mathrm{E}-08 & 2.08930 \mathrm{E}-08 & 2.18776 \mathrm{E}-08 & 2.29087 \mathrm{E}-08 & 2.39883 \mathrm{E}-08\end{array}$ 2.51189E-08 2.63027E-08 2.75423E-08 2.88403E-08 3.01995E-08 3.16228E-08 3.31131E- 08 3.46737E- 08 3.63078E- 08 3.80189E- 08 3. $98107 \mathrm{E}-08$ 4.16869E- 08 4.36516E- 08 4.57088E- 08 4.78630E- 08 5. $01187 \mathrm{E}-08$ 5.24807E-08 5.49541E-08 5.75440E-08 $\quad 6.02560 \mathrm{E}-08$ 6.30957E-08 6.60693E-08 6.91831E-08 7.24436E-08 7.58578E-08
} 
7.94328E-๑8 8.31764E-๑8 8.70964E-๑8 9.12011E- 08 9.54993E-๑8 1. $00000 \mathrm{E}-07$ 1.04713E-07 1.09648E- 07 1.14815E- 07 1.20226E- 07 $1.25893 \mathrm{E}-07$ 1.31826E- 07 1.38038E- 07 1.44544E- 07 1.51356E- 07 $1.58489 \mathrm{E}-07$ 1.65959E-07 1.73780E- 07 1.81970E- 07 1.90546E- 07 $\begin{array}{lllll}1.99526 E-07 & 2.08930 E-07 & 2.18776 E-07 & 2.29087 E-07 & 2.39883 E-07\end{array}$ 2.51189E-07 2.63027E-07 2.75423E-07 2.88403E-07 3.01995E-07 3.16228E-07 3.31131E- 07 3.46737E-07 3.63078E- 07 3.80189E- 07 3.98107E-07 4.16869E- $07 \quad 4.36516 E-07 \quad 4.57088 E-07 \quad 4.78630 E-07$ 5. $01187 \mathrm{E}-07$ 5.24807E-07 5.49541E-07 5.75440E-07 6. $\quad$ 52560E-07 6.30957E-07 6.60693E-07 6.91831E- 07 7.24436E-07 7.58578E- 07 7.94328E- 07 8.31764E-07 8.70964E- 07 9.12011E- 07 9.54993E- 07 $1.00000 \mathrm{E}-06$ 1.04713E-06 1.09648E-06 1.14815E-06 $1.20226 \mathrm{E}-06$ $1.25893 \mathrm{E}-06$ 1.31826E-@6 1.38038E- 06 1.44544E- 06 1.51356E- 06 1.58489E-@6 1.65959E-@6 1.73780E-06 1.81970E-06 1.90546E-06 1.99526E-06 2.08930E-06 2.18776E-06 2.29087E-06 2.39883E-06 2.51189E-06 2.63027E-06 2.75423E-06 2.88403E-06 3.01995E-06 3.16228E-06 3.31131E-06 3.46737E-06 3.63078E-06 3.80189E-06 3. $98107 \mathrm{E}-06$ 4.16869E-@6 4.36516E- 06 4.57088E- 06 4.78630E- 06 5.01187E-06 5.24807E-06 5.49541E-06 5.75440E-06 6.02560E-06 6.30957E-06 6.60693E-06 6.91831E-06 7.24436E-06 7.58578E-06 7.94328E-06 8.31764E-@6 8.70964E-06 9.12011E-06 9.54993E-06 1.00000E-05 1.04713E-05 1.09648E-05 1.14815E-05 1.20226E-05 $1.25893 \mathrm{E}-05$ 1.31826E- 05 1.38038E- 05 1.44544E- 05 1.51356E- 05 1.58489E-05 1.65959E-05 1.73780E-05 1.81970E-05 1.90546E-05 1.99526E-05 2.08930E-05 2.18776E-05 2.29087E-05 2.39883E-05 2.51189E-05 2.63027E-05 2.75423E-05 2.88403E-05 3.01995E-05 3.16228E-05 3.31131E-05 3.46737E-05 3.63078E-05 3.80189E-05 3.98107E-05 4.16869E-05 4.36516E-05 4.57088E-05 4.78630E-05 5.01187E-05 5.24807E-05 5.49541E-05 5.75440E-05 6.02560E-05 6.30957E-05 6.60693E-05 6.91831E-05 7.24436E-05 7.58578E-05 7.94328E-05 8.31764E-05 8.70964E-05 9.12011E-05 9.54993E-05 1. $00000 \mathrm{E}-\odot 4$ 1.04713E-๑4 1.09648E-๑4 1.14815E-04 1.20226E-๑4 $1.25893 \mathrm{E}-04$ 1.31826E-04 1.38038E-04 1.44544E-04 1.51356E-04 $1.58489 E-04$ 1.65959E-@4 1.73780E-@4 1.81970E- 04 1.90546E- 04 1.99526E-04 2.08930E-04 2.18776E-04 2.29087E-04 2.39883E-04 2.51189E-04 2.63027E-04 2.75423E-04 2.88403E-04 3.01995E-04 3.16228E-04 3.31131E-04 3.46737E-04 3.63078E-04 3.80189E-04 3. $98107 \mathrm{E}-04$ 4.16869E-๑4 4.36516E- 04 4.57088E- $04 \quad 4.78630 \mathrm{E}-04$ 5. $01187 \mathrm{E}-04$ 5.24807E-@4 5.49541E-@4 5.75440E-04 6.02560E-@4 6.30957E-@4 6.60693E-@4 6.91831E- 04 7.24436E-04 7.58578E-04 7.94328E-๑4 8.31764E-๑4 8.70964E-๑4 9.12011E-04 9.54993E-๑4 1. $00000 \mathrm{E}-03$ 1.04713E-03 1.09648E-03 1.14815E-03 1.20226E-03

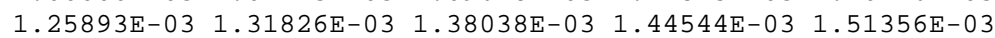

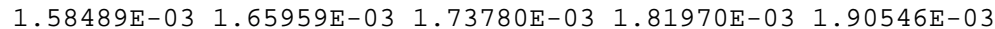

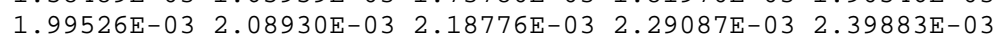
2.51189E-03 2.63027E-03 2.75423E-03 2.88403E-03 3.01995E-03

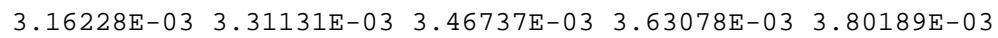
3. 98107E-03 4.16869E-03 4.36516E-03 4.57088E-03 4.78630E-03 5. 01187E-03 5.24807E-03 5.49541E-03 5.75440E-03 6.02560E-03 6.30957E-๑3 6.60693E-@3 6.91831E-@3 7.24436E-@3 7.58578E-03 7.94328E-03 8.31764E-03 8.70964E-03 9.12011E-03 9.54993E-03

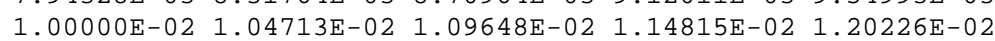
1.25893E-02 1.31826E-02 1.38038E-02 1.44544E-02 1.51356E-02 $1.58489 \mathrm{E}-02$ 1.65959E-02 1.73780E-๑2 1.81970E-02 1.90546E-02 1.99526E-@2 2.08930E-02 2.18776E-02 2.29087E-02 2.39883E-02 2.51189E-02 2.63027E-02 2.75423E-02 2.88403E-02 3.01995E-02 3.16228E-02 3.31131E-02 3.46737E-02 3.63078E-02 3.80189E-02 3.98107E-02 4.16869E-02 4.36516E-02 4.57088E-02 4.78630E-02 5. $01187 \mathrm{E}-02$ 5.24807E-02 5.49541E-02 5.75440E-02 6.02560E-02 6.30957E-02 6.60693E-02 6.91831E-02 7.24436E-02 7.58578E-02 7.94328E-02 8.31764E-02 8.70964E-02 9.12011E-02 9.54993E-02 1.000๑९E-01 1.04713E-01 1.09648E-01 1.14815E-01 1.20226E-01 $1.25893 \mathrm{E}-01$ 1.31826E-01 1.38038E- 01 1.44544E-01 1.51356E-01 1.58489E-01 1.65959E-01 1.73780E-01 1.81970E-01 1.90546E-01

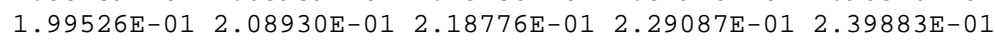
2.51189E-01 2.63027E-01 2.75423E-01 2.88403E-01 3.01995E-01 3.16228E-01 3.31131E-01 3.46737E-01 3.63078E-01 3.80189E-01 3.98107E-01 4.16869E-01 4.36516E-01 4.57088E-01 4.78630E-01 5. $01187 \mathrm{E}-01$ 5.24807E-01 5.49541E-01 5.75440E-01 6.02560E-01 6.30957E-01 6.60693E-01 6.91831E-01 7.24436E-01 7.58578E-01 7.94328E-01 8.31764E-01 8.70964E-01 9.12011E-01 9.54993E-01 


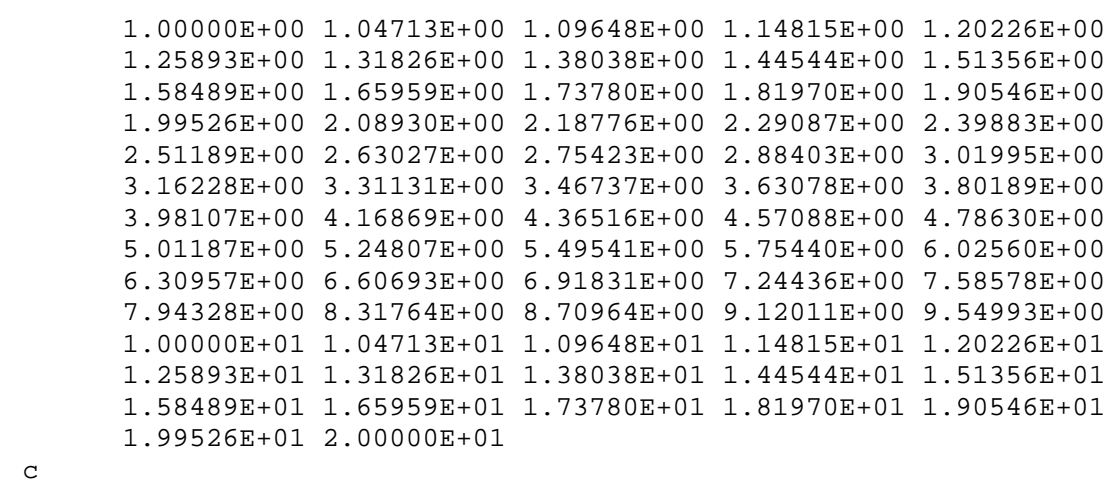

\section{Definition of K-eff}

For time independent codes there is a very simple textbook definition that can be used to define K-eff. It is the ratio of the number of neutrons produced by fission in one generation to the number produced in the preceding generation; these codes need not consider anything else. For time dependent codes or codes that define K-eff in terms of a balance between neutrons produced and removed this is more complicated, because fission is not the only process that can produce neutrons during a generation; there is also $(n, 2 n),(n, 3 n)$, etc., and how codes handle these lead to different definition of K-eff. Below I'll explain the differences.

Starting from the time dependent, linear Boltzmann equation in general geometry,

$$
\frac{1}{v} \frac{\partial N}{\partial t}+\Omega^{*} \nabla N+\Sigma t^{*} N=\iint(<v>\Sigma f+\Sigma \text { scatter }+2 \Sigma n, 2 n+3 \Sigma n, 3 n+\ldots \ldots) N d \Omega^{\prime} d E^{\prime}
$$

Where $N(r, \Omega, E, t)$ is the neutron flux, $v^{*} n(r, \Omega, E, t), v$ is the neutron speed, $\Sigma t$ is the macroscopic total cross section, $\langle v\rangle$ is the average number of neutrons emitted per fission, $\Sigma f$, $\Sigma$ scatter , $\Sigma n, 2 n, \Sigma n, 3 n$, etc., are the macroscopic cross sections for each type of event. For simplicity I will use neutron density $n(r, \Omega, E, t)$ in the following,

Integrate over all space, energy, and direction

$$
\frac{\partial n}{\partial t}+[L * v * n]+[\Sigma t * v * n]=[(<v>\Sigma f+\Sigma s c a t t e r+2 \Sigma n, 2 n+3 \Sigma n, 3 n+\ldots . .) v * n]
$$

Collecting terms together we find a simple equation defining the time dependent behavior of the system,

$$
\begin{aligned}
& \frac{\partial n}{\partial t}=\alpha^{*} n \\
& \alpha=[(<v>\Sigma f+\Sigma \text { scatter }+2 \Sigma n, 2 n+3 \Sigma n, 3 n+\ldots . .) v]-[L * v]-[\Sigma t * v]
\end{aligned}
$$


$=[$ Production rate $]-[$ Removal Rate $]$

The time constant $(\alpha)$ is a physical observable and as such has a unique value that we can determine. The non-uniqueless of K-eff and related terms is because exactly the same terms appear in this definition of $\alpha$ as positive and negative terms that we can completely cancel (scatter), or as simply related terms that we can partially cancel (n,2n).

I will divide the total cross section by events according to how many neutrons result from each type of event: none - capture, (n,p), (n,a), etc., one - scatter, (n,np), (n,na), etc., more than one - fission, $(n, 2 n),(n, 3 n)$, etc.. All of those events that result in one neutron do not directly effect the neutron balance of the system (they effect it indirectly through the leakage), and appear in exactly the same form in this definition as positive and negative terms, so that we can cancel them. Upon cancelling scatter, (n,np), (n,na), etc.,

$\alpha=[(<v>\Sigma f+2 \Sigma n, 2 n+3 \Sigma n, 3 n+\ldots .) v]-.[L * v]-[(\Sigma n, 0+\Sigma f+\Sigma n, 2 n+\Sigma n, 3 n+\ldots) * v]$

Up to this point all or least most of the codes use the same definitions, and this is the definition that TART uses, i.e., any event that introduces additional neutrons into the system is considered production, and any event that produces neutrons also removes neutrons, etc., $(\mathrm{n}, 2 \mathrm{n})$ removes one neutron and produces two neutrons,

Production rate $=[(<v>\Sigma f+2 \Sigma n, 2 n+3 \Sigma n, 3 n+\ldots .) v$.

Removal Rate $=$ Leakage + Absorption $=\left[L^{*} v\right]+[(\Sigma n, 0+\Sigma f+\Sigma n, 2 n+\Sigma n, 3 n+\ldots) * v]$

Other codes change this to agree with the textbook definition of K-eff where production is only due to fission. This requires them to subtract $2 \Sigma n, 2 n+3 \Sigma n, 3 n+.$. from the production and removal resulting in the definitions,

Production rate $=[(<v>\Sigma f) v]$

Removal Rate $=\left[L^{*} v\right]+[(\Sigma n, 0+\Sigma f-\Sigma n, 2 n-2 \Sigma n, 3 n-3 \Sigma n, 4 n-\ldots) * v]$

Note, that we still have exactly the same definition of the physically observable time constant $(\alpha)$, and for an exactly critical system K-eff remains unity using any of these definitions. Regardless of how they define production and removal, the codes define,

$\alpha=[$ Production Rate $]-[$ Removal Rate $]=\left[\frac{\text { Production }}{\text { Re moval }}-1\right]^{*}[$ Removal Rate $]$

$$
=[\mathrm{K} \text {-eff }-1] / \operatorname{Tr} \quad \operatorname{Tr}=\text { Removal Time }
$$

K-eff $=\frac{\text { Production }}{\text { Re } \text { moval }} \quad$ Removal Time $=1 /[$ Removal Rate $]$

Here we can see that even though the time constant $(\alpha)$ has a unque definition, K-eff and the removal time, do not, since all codes do not define production and removal the same 
way. With the TART definition any event that produces more than one neutron ends a generation, and adds to the removal $\Sigma f+\Sigma n, 2 n+\Sigma n, 3 n+\ldots$ and adds to the production $<v>\Sigma f+2 \Sigma n, 2 n+3 \Sigma n, 3 n+\ldots .$. Codes that do not consider that $(n, 2 n),(n, 3 n)$, etc., end a generation, add nothing to production for these events and subtract from the removal $\Sigma n, 2 n+2 \Sigma n, 3 n+3 \Sigma n, 4 n+\ldots$.

As a quantitative example by Andrej Trkov for WIMS, the K-eff from codes, which adopt the convention of defining production from fission alone require a correction, which is easily implemented for the "no leakage" case. We must add $2 \Sigma n, 2 n+3 \Sigma n, 3 n+$.. to the production and removal and using the absorption as a normalization results in the definitions,

$\mathrm{K}$-eff $=\left(\mathrm{K}-\mathrm{eff}^{*}+2 \Sigma n, 2 n / \Sigma a+3 \Sigma n, 3 n / \Sigma a+\ldots\right) /(1+2 \Sigma n, 2 n / \Sigma a+3 \Sigma n, 3 n / \Sigma a+\ldots)$,

Here $\Sigma a$ is the absorption cross section (including fission). The ratios $\Sigma n, 2 n / \Sigma a$ calculated with WIMS-D for the three cases are:

$1 / 2 ” \quad 0.00182$

$1 / 4 " \quad 0.00083$

$1 / 8 ” \quad 0.00032$

From the above it follows that the correction amounts to 0.00015 for the $1 / 2$ " bound case and smaller for all other cases; in other words, it is well below the integral differences we see I this study, and yet important when we try to compare energy dependent spectra. It is worth noting that even in the case of the very thermalized systems, because fission at any energy creates fission neutrons above the $(n, 2 n)$ threshold, $(n, 2 n)$ accounts for a few tenths of a per-cent of the total neutrons produced in these systems; it is even higher for faster neutron systems. Therefore in terms of the overall neutron economy it is not an effect that can be simply ignored.

\section{Where do we go from here: Proposed Future Detailed Comparisons}

I propose that in the future we perform more detailed comparisons, including the production, absorption, and leakage energy dependent spectra. In order to do this we all need to use the same definitions.

Any of the above definitions is physically acceptable, and in most situations the calculated integral parameters, such as K-eff are very similar, but differences do create a problem if we are to compare production, absorption and leakage energy dependent spectra.

For comparison of spectra I propose that we use the TART definition to include all neutrons introduced into the system as production, and all neutrons that are absorbed or leaked as removal. In which case $(n, 2 n),(n, 3 n)$, etc., make positive contributions to both production and removal. For our comparisons tally the following quantities at the energies at which the event happened, not the energy at which neutrons were 
subsequently are emitted. For example, for fission tally one neutron removal and $\langle v\rangle$ neutrons produced at the energy where the fission was induced, not the fission emission spectrum in which the neutrons are emitted; the former is very specific for each system and will provide us with the information that we need, the latter will not.

Production $=<v>\Sigma f+2 \Sigma n, 2 n+3 \Sigma n, 3 n+\ldots$.

Absorption $=\Sigma n, 0+\Sigma f+\Sigma n, 2 n+\Sigma n, 3 n+\ldots$

Leakage $=L$

These quantities are integrated over the entire system, such that if we further integrate the spectra over energy we should be able to exactly reproduce K-eff,

K-eff $=[$ Production $] /[$ Absorption + Leakage $]$

Tally from $1.0 \mathrm{~d}-11 \mathrm{MeV}$ to $20 \mathrm{MeV}$ using the below listed 616 energy tally bin structure, or a more efficient method is to directly define tally bins. The definition of the tally bin index is trivial, since there are 50 tally bins per energy decade, uniformly spaced in lethargy (log of energy),

$$
\begin{aligned}
\text { Index } & =0<1.0 \mathrm{~d}-11 \mathrm{MeV} \\
& =1 \text { to } 616: 1.0 \mathrm{~d}-11 \mathrm{MeV} \text { to } 20 \mathrm{MeV}=1+50 * \operatorname{alog} 10(\mathrm{E} / 1.0 \mathrm{~d}-11) ; \mathrm{E} \text { in } \mathrm{MeV} \\
& =617>20 \mathrm{MeV}
\end{aligned}
$$

So that in the future we can compare code results in greater detail, if any code designer adds these quantities to the output of their code in any format that they want, I (Red Cullen) will "volunteer" to write a utility code to put your results into a standard form for comparison to the detailed output from other codes. We already have a number of codes which are connected in this manner, and our results are providing a great deal of insight and understanding to the differences we see in the output from a variety of codes.

As an example of how much we can learn from detail comparisons I selected two codes that produce very similar results for simple integral parameters such as K-eff for our pin cell problems. Below I show a detailed comparison of the neutron production spectra. In the first figure we can see excellent agreement over most of the energy range, except for at very low energy. The second figure shows a detail of the very low energy, and from this can can see that one code is modfelling the thermal scatteriong law continuous in secondary energy, whereas the other is modelling it as a series of discrete secondary energies for hydrogen bound in water. The only reason that this second spectrum does not drop lower between the discrete energies, is because of free atom scattering in oxygen and to a lesser degree uranium. 


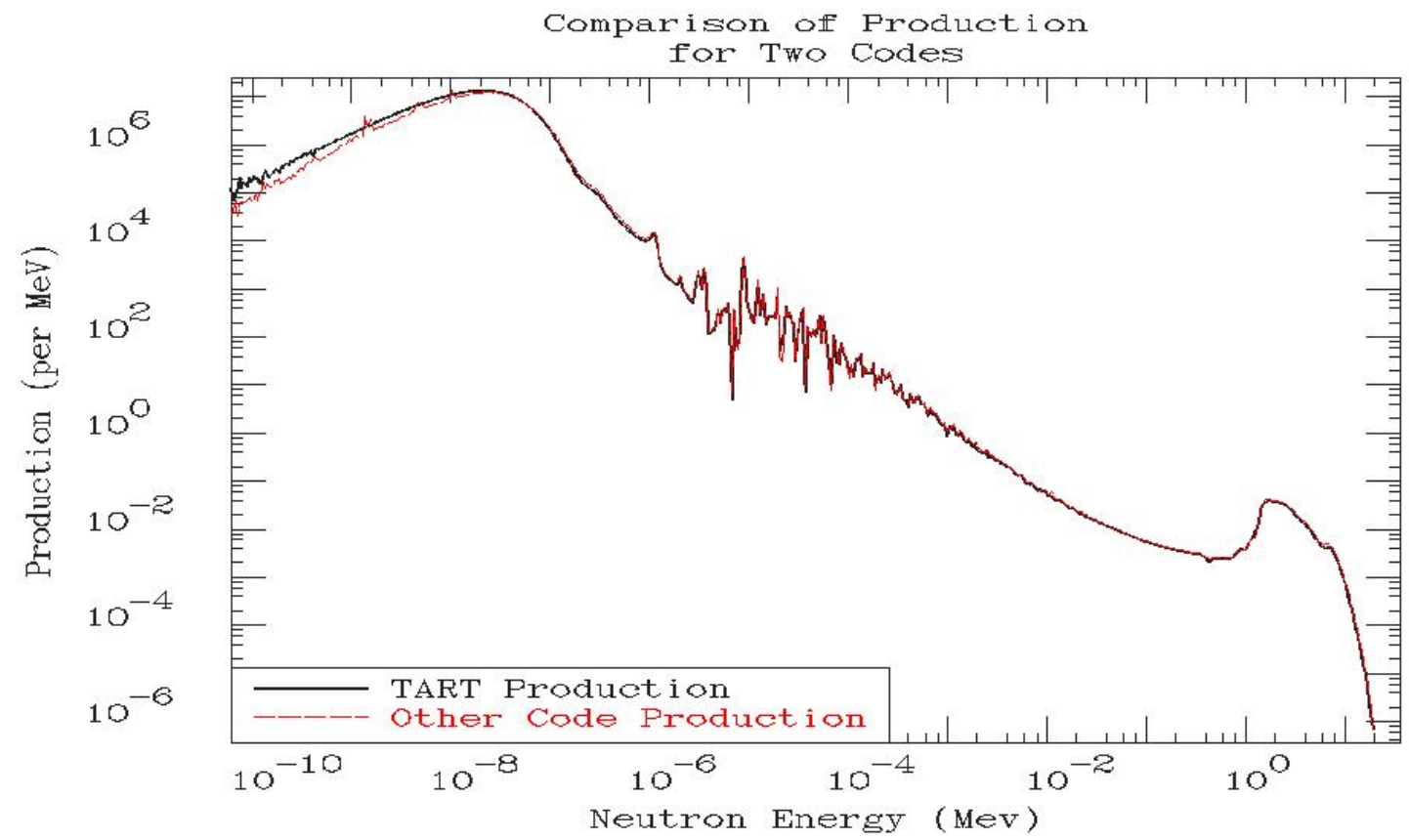

Fig. 17: Comparison of Code Results

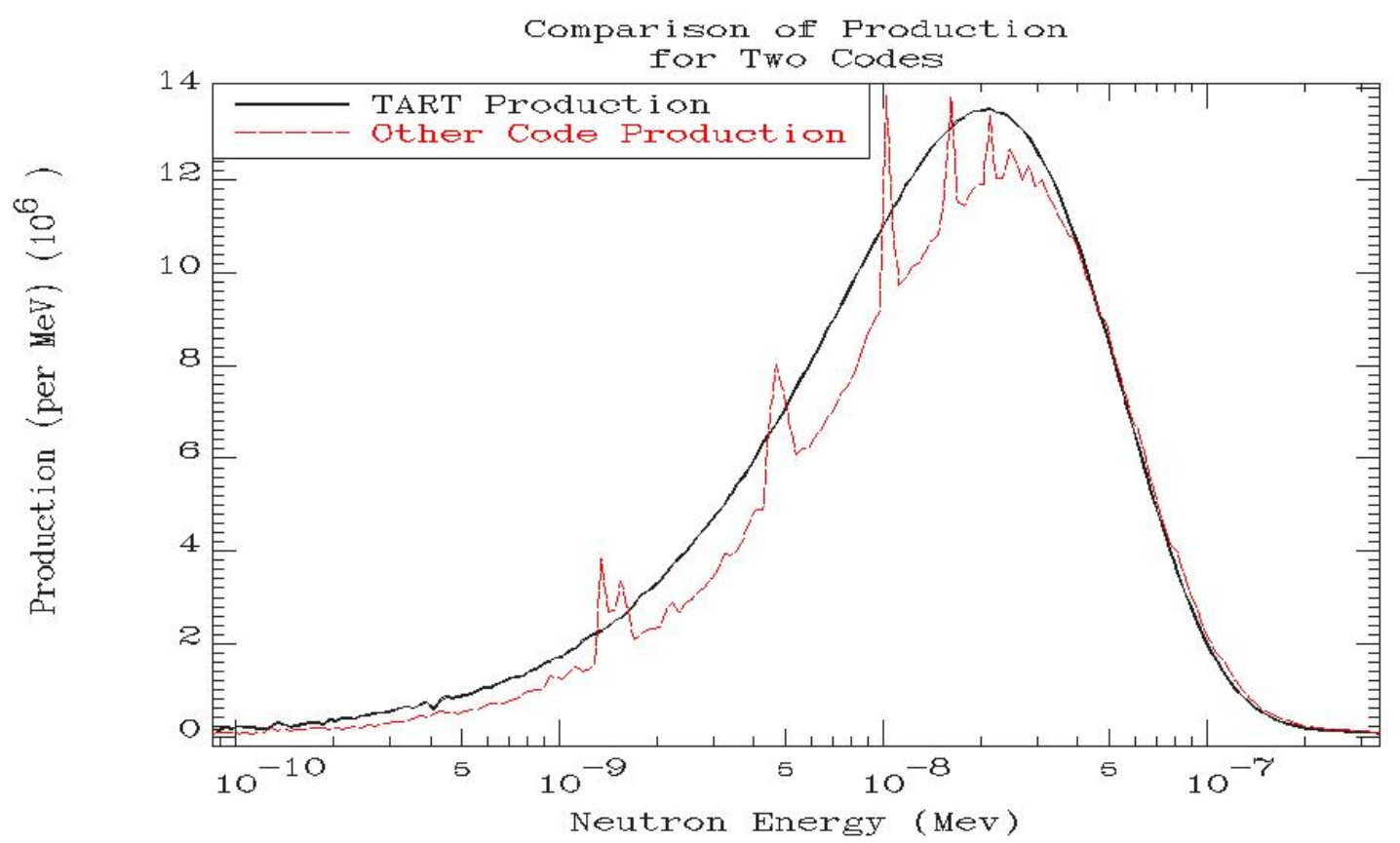

Fig. 18: Detail of Comparison of Code Results 
University of California

Lawrence Livermore National Laboratory

Technical Information Department

Livermore, CA 94551

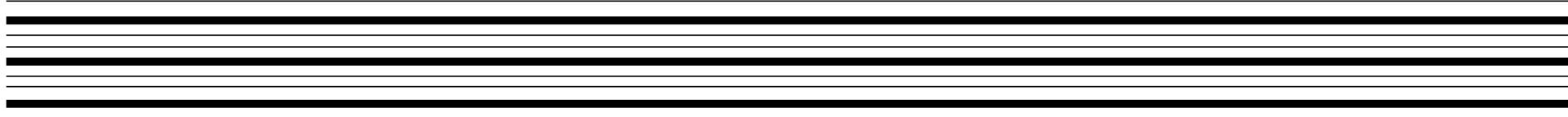

